\title{
Ergodic properties of continuous-time random walks: finite-size effects and ensemble dependences
}

\author{
Tomoshige Miyaguchi ${ }^{1, *}$ and Takuma Akimoto ${ }^{2, \dagger}$ \\ ${ }^{1}$ Department of Mathematics Education, Naruto University of Education, Tokushima 772-8502, Japan \\ ${ }^{2}$ Department of Mechanical Engineering, Keio University, Yokohama 223-8522, Japan
}

(Dated: November 5, 2018)

\begin{abstract}
The effects of spatial confinements and smooth cutoffs of the waiting time distribution in continuous-time random walks (CTRWs) are studied analytically. We also investigate dependences of ergodic properties on initial ensembles (i.e., distributions of the first waiting time). Here, we consider two ensembles: the equilibrium and a typical non-equilibrium ensembles. For both ensembles, it is shown that the time-averaged mean square displacement (TAMSD) exhibits a crossover from normal to anomalous diffusion due to the spacial confinement and this crossover does not vanish even in the long measurement time limit. Moreover, for the non-equilibrium ensemble, we show that the probability density function of the diffusion constant of TAMSD follows the transient MittagLeffler distribution, and that scatter in the TAMSD shows a clear transition from weak ergodicity breaking (an irreproducible regime) to ordinary ergodic behavior (a reproducible regime) as the measurement time increases. This convergence to ordinary ergodicity requires a long measurement time compared to common distributions such as the exponential distribution; in other words, the weak ergodicity breaking persists for a long time. In addition, it is shown that, besides the TAMSD, a class of observables also exhibits this slow convergence to ergodicity. We also point out that, even though the system with the equilibrium initial ensemble shows no aging, its behavior is quite similar to that for the non-equilibrium ensemble.
\end{abstract}

PACS numbers: 05.40.Fb, 02.50.Ey, 87.15.Vv

\section{INTRODUCTION}

Recently, slow anomalous diffusion, which is defined by the sublinear dependence of the mean square displacement (MSD) on time, has been found in various phenomena including lipid granules diffusing in living fission yeast cells $[1,2]$, colloidal particles diffusing on sticky surfaces [3] and in networks of entangled actin filaments [4], mRNA molecules diffusing in E. coli [5], chromosomal loci diffusing in bacteria [6], telomeres diffusing in nuclei of eukaryote cells [7], and proteins diffusing in dextran solutions [8]. To understand these phenomena, two types of slow diffusion models have been extensively studied so far: (1) continuous-time random walks (CTRWs) [1-4] and (2) the generalized Langevin equation (GLE) and fractional Brownian motions (FBMs) [59]. For the CTRW model, if the probability density function (PDF) of the waiting times between jumps of the particle is a power law $w(\tau) \sim 1 / \tau^{1+\alpha}$ with $0<\alpha<1$, the ensemble-averaged MSD (EAMSD) shows slow diffusion [10, 11]: $\left\langle(\delta x)^{2}\right\rangle \sim t^{\alpha}[12]$. Typical origins of such power-law waiting times are complex energy landscapes $[3,11,13-15]$ and diffusion in inner degrees of freedom $[16,17]$. Similarly, EAMSD of GLE shows slow diffusion if the memory kernel $\eta(t)$ decays algebraically $\eta(t) \sim 1 / t^{\alpha}$ with $0<\alpha<1[18,19]$. A possible origin of this non-Markovian memory effect is the viscoelasticity of the medium [20].

\footnotetext{
*tmiyaguchi@naruto-u.ac.jp

† akimoto@z8.keio.jp
}

A primary difference between these two stochastic processes - CTRWs and GLE - is in their ergodic properties. Even if the memory kernel of a GLE is given by a power function as stated above, the GLE satisfies the ergodic property, that is, time-averaged quantities coincide with ensemble-averaged quantities [21, 22]. In contrast, this equivalence between the two averages does not hold in the CTRWs with power-law waiting times. However, such CTRWs exhibit an extended form of ergodicity - time-averaged quantities become random variables following a distribution function $[15,23]$. This distributional ergodicity is called weak ergodicity breaking $[15,23-27]$ or infinite ergodicity $[28,29]$.

In addition to the above mentioned examples, CTRWs are frequently used in various fields of science $[10,11,30$, 31]. However, there are cases in which some finite-size effects should be considered in order to compare the model with experimental data. In particular, cutoffs at the tail of the power-law waiting time distribution $[3,13,31]$ and spatial confinement effects $[1,2]$ arise in many systems. The typical origins of waiting time cutoffs are physical limits with respect to energy and spatial extensions (e.g., $[3,11,13,32])$. Theoretical analysis of the CTRWs with such cutoffs is difficult, because it is necessary to investigate the transient behavior. However, this difficulty is avoidable by using the tempered stable distribution (TSD) $P_{\mathrm{TL}}(\tau, \lambda)[22,23,33]$. The TSD has exponentially smooth cutoffs, and is a modified version of the truncated stable distribution, which has a sharp cutoff at the tail [34]. Furthermore, the TSD has the property called infinite divisibility [22, 33, 35-37], which allows a rigorous analysis even for transient behavior. The TSD is a spe- 
cial model for the cutoffs, but it shows typical behavior of cutoff distributions such as a slow convergence to the Gaussian distribution [22, 33, 35-37]. Furthermore, the generalized fractional Fokker-Planck equation (GFFPE), which we will use in this paper to study EAMSD, has been derived for CTRWs with tempered stable waiting times [33]. The ergodic properties of the system without confinement have also been clarified in [23], where the existence of a clear transition from weak ergodicity breaking (an irreproducible regime) to ordinary ergodicity (a reproducible regime) was shown analytically for the case of a non-equilibrium initial ensemble (See Sec. II for a precise definition of the non-equilibrium and equilibrium ensembles).

Another important finite-size effect is spatial confinements. For example, to understand the transport phenomena in cells [5], confinements due to cell membranes should be considered. Confinement effects in CTRWs with power-law waiting times were studied numerically in [26] and analytically in [38]; it was found that the timeaveraged MSD (TAMSD) shows a crossover from normal diffusion at short timescales to anomalous slow diffusion at longer timescales. They also reported numerically the weak ergodicity breaking in TAMSD.

Recently, the CTRW model with the two finite-size effects (i.e., waiting time cutoffs and spatial confinements) has been used as a model for the transport of lipid granules in living fission yeast cells $[1,2]$, in which confinement effects are caused by a Hookean force exerted by optical tweezers. The model clearly explains the experimental results such as the crossover in TAMSD and weak ergodicity breaking. These studies mainly used numerical simulations, but a detailed theoretical analysis has not yet been reported. Also, they studied only nonequilibrium ensemble, and thus the dependences of the ergodic properties on initial ensembles are still unknown.

In this paper, we present theoretical results for CTRWs with two finite-size effects: the effects of cutoffs in the waiting time distribution and the confinement effects. In particular, we focus on TAMSD as an observable, and study the crossover from normal to anomalous diffusion in the TAMSD and ergodic properties in terms of the scatter of diffusion constant for the TAMSD. The TAMSD, which is often used in single-molecule tracking experiments $[1,2,5,7,20,39-41]$, is defined as

$$
\overline{(\delta x)^{2}}(\Delta, t) \equiv \frac{1}{t-\Delta} \int_{0}^{t-\Delta}\left|x\left(t^{\prime}+\Delta\right)-x\left(t^{\prime}\right)\right|^{2} d t^{\prime}
$$

where $x\left(t^{\prime}\right)$ is the position of the particle at time $t^{\prime}, t$ is the total measurement time, and $\Delta$ is the time interval. Hereinafter we assume that $\Delta \ll t$. Here we define a generalized diffusion constant $D$ as $\overline{(\delta x)^{2}}(\Delta, t) \simeq D \Delta^{\beta}$. In some experiments, it has been reported that $D$ behaves like a random variable depending on each time series $[1$, $2,5,7,39]$. Therefore, the scatter in TAMSD or $D$ has been used to check the consistency of the model with experimental data $[1,2,42]$.
In this paper, we use the TSDs $[22,33,35-37]$ as waiting time distributions of CTRWs. For the TSD, it is possible to explicitly write the convoluted waiting distributions of any order [Eq. (5)]. Moreover, we use the numerical method for the TSD presented in [33] and Appendix B, and study the initial and boundary value problem of the GFFPE to understand the confinement effect.

The rest of this paper is organized as follows. In Sec. II, we introduce the TSDs. Then, in Sec. III, we show the crossover from normal to anomalous diffusion in TAMSD by using the GFFPE. In Sec. IV, ergodic properties of TAMSD are studied using renewal theoretic analysis. Secs. V and VI are devoted to conclusion and discussion. In the appendices, we summarize some technical matters, including a derivation of GFFPE from CTRWs as well as the ergodic properties of general observables.

\section{TEMPERED STABLE DISTRIBUTION}

In this paper, we consider CTRWs confined in a one-dimensional lattice with unit lattice constant: $(1,2, \ldots, L)$. Jumps are permitted only to the nearest neighbor sites without preferences, although this can be generalized to jump length distributions with zero mean and finite variances. It is also assumed that the particle is reflected if it goes beyond the permitted region (for example, if the particle jumps into the site $L+1$, it is pushed back to $L$ ). This system is a continuous-time version of the discrete-time random walks (DTRWs) with reflecting barriers [43].

Furthermore, successive waiting times of the particle $\tau_{k}(k=1,2, \ldots)$ between jumps are assumed to be mutually independent and follow the $\operatorname{TSD} P_{\mathrm{TL}}\left(\tau_{k}, \lambda\right)$. The Laplace transform of the TSD, $\tilde{P}_{\mathrm{TL}}(s, \lambda) \equiv$ $\int_{0}^{\infty} d \tau e^{-s \tau} P_{\mathrm{TL}}(\tau, \lambda)$, is given by

$$
\tilde{P}_{\mathrm{TL}}(s, \lambda)=\exp \left(-c\left[(\lambda+s)^{\alpha}-\lambda^{\alpha}\right]\right),
$$

where $\alpha \in(0,1)$ is the stable index, $c$ is a scale factor, and $\lambda \geq 0$ is a parameter characterizing the smooth cutoff. Note that when $\lambda=0$, this is the Laplace transform of the one-sided stable distributions. Equivalently, the characteristic function of the TSD, $e^{\psi(s, \lambda)} \equiv$ $\int_{-\infty}^{\infty} d \tau P_{\mathrm{TL}}(\tau, \lambda) e^{i \zeta \tau}$, is given by

$$
e^{\psi(\zeta, \lambda)}=\exp \left(-c\left[(\lambda-i \zeta)^{\alpha}-\lambda^{\alpha}\right]\right),
$$

This characteristic function is a special case of the TSD given in $[22,33,35-37]$. This is because $\tau$ takes only positive values, and we need only one-sided distributions. More precise definition of the TSD and the derivations of the above equations are presented in Appendix A.

The TSD in real space is also derived explicitly as follows (for a derivation, see Appendix A):

$$
\begin{aligned}
P_{\mathrm{TL}}(\tau, \lambda)= & -\frac{e^{c \lambda^{\alpha}-\lambda \tau}}{\pi \tau} \\
& \times \sum_{k=1}^{\infty} \frac{\Gamma(k \alpha+1)}{k !}\left(-c \tau^{-\alpha}\right)^{k} \sin (\pi k \alpha),(4)
\end{aligned}
$$


where $\Gamma(x)$ is the gamma function. When $\lambda=0$, $P_{\mathrm{TL}}(\tau, 0)$ is the one-sided $\alpha$-stable distribution with a power-law tail: $P_{\mathrm{TL}}(\tau, 0) \sim 1 / \tau^{1+\alpha}$ as $\tau \rightarrow \infty$ [44, 45]. Therefore, the TSD [Eq. (4)] is the one-sided stable distribution multiplied by the exponential function $e^{-\lambda \tau}$ : $P_{\mathrm{TL}}(\tau, \lambda) \propto e^{-\lambda \tau} P_{\mathrm{TL}}(\tau, 0)$. Thus, $P_{\mathrm{TL}}(\tau, \lambda)$ behaves as $P_{\mathrm{TL}}(\tau, \lambda) \sim e^{-\lambda \tau} / \tau^{1+\alpha}$ when $\tau \rightarrow \infty$.

Moreover, the $n$-times convoluted $\operatorname{PDF} P_{\mathrm{TL}}^{n}(\tau, \lambda)$, which is the PDF of the sum of the successive waiting times $t_{n} \equiv \sum_{k=1}^{n} \tau_{k}$, is expressed by using $P_{\mathrm{TL}}(\tau, \lambda)$ :

$$
P_{\mathrm{TL}}^{n}(\tau, \lambda)=n^{-1 / \alpha} P_{\mathrm{TL}}\left(n^{-1 / \alpha} \tau, n^{1 / \alpha} \lambda\right) .
$$

Therefore, the $n$-times convoluted $\operatorname{PDF} P_{\mathrm{TL}}^{n}(\tau, \lambda)$ is also explicitly derived from Eqs. (4) and (5). Using Eq. (5), we derive transient properties of CTRWs, including various crossovers, in the following sections.

Even though we use the TSD $P_{\mathrm{TL}}(\tau, \lambda)$ as a waiting time distribution, we should further specify the first waiting time $\tau_{1}$, or equivalently, the initial ensemble (Here, the first waiting time $\tau_{1}$ is the time interval between the start of the measurement and the first jump. We always assume that measurements start at $t^{\prime}=0$.). In this paper, we consider two kinds of initial ensembles. The first one is a typical and most frequently used non-equilibrium ensemble, for which the first waiting times $\tau_{1}$ of the particles are chosen from $P_{\mathrm{TL}}(\tau, \lambda)$. The second one is the equilibrium ensemble, for which the first waiting times $\tau_{1}$ are chosen from the equilibrium waiting time distribution $P_{\mathrm{TL}}^{\mathrm{eq}}(\tau, \lambda)$. Here, $P_{\mathrm{TL}}^{\mathrm{eq}}(\tau, \lambda)$ can be defined by its Laplace transformation $[46,47]$ :

$$
\tilde{P}_{\mathrm{TL}}^{\mathrm{eq}}(s, \lambda)=\frac{1-\tilde{P}_{\mathrm{TL}}(s, \lambda)}{\langle\tau\rangle s},
$$

where $\langle\tau\rangle=c \lambda^{\alpha-1} \alpha$ is the mean waiting time for $P_{\mathrm{TL}}(\tau, \lambda)$. Note that the second and subsequent waiting times, $\tau_{2}, \tau_{3}, \ldots$, are chosen from $P_{\mathrm{TL}}(\tau, \lambda)$ for both ensembles. Numerical methods to generate random variables following $P_{\mathrm{TL}}(\tau, \lambda)$ and $P_{\mathrm{TL}}^{\mathrm{eq}}(\tau, \lambda)$ are summarized in Appendix B. $P_{\mathrm{TL}}^{\mathrm{eq}}(\tau, \lambda)$ can be expressed analytically as follows

$$
\begin{aligned}
P_{\mathrm{TL}}^{\mathrm{eq}}(\tau, \lambda) & =-\frac{e^{c \lambda^{\alpha}}}{\pi\langle\tau\rangle} \\
& \times \sum_{k=1}^{\infty} \frac{\Gamma(k \alpha+1)}{k !}\left(-c \tau^{-\alpha}\right)^{k} \sin (\pi k \alpha) f_{k}(\tau),
\end{aligned}
$$

where $f_{k}(\tau)$ is defined by $f_{k}(\tau)=\int_{0}^{1} e^{-\lambda \tau / a} a^{\alpha k-1} d a$. See Appendix A for a derivation of Eq. (7). The mean waiting time for $P_{\mathrm{TL}}^{\mathrm{eq}}(\tau, \lambda)$ is given by $\langle\tau\rangle_{\mathrm{eq}}=\left(c \lambda^{\alpha} \alpha+1-\right.$ $\alpha) /(2 \lambda) \simeq(1-\alpha) /(2 \lambda)$, which is much longer than $\langle\tau\rangle$ if $\lambda$ is small.

\section{ENSEMBLE AVERAGE OF TAMSD}

The confinement effects on CTRWs with power-law waiting times were investigated in [38] by using the Fractional Fokker-Planck equation (FFPE). Here instead of the FFPE, we use GFFPE, which was derived in [33], to incorporate the smooth cutoff into the waiting time distribution and to study the ensemble averages of the TAMSD.

There are three timescales in the present model: (1) time interval $\Delta,(2)$ total measurement time $t$, and (3) timescale of the cutoff $1 / \lambda$. Let us define the Laplace variables $u$ and $s$ conjugate to $\Delta$ and $t$, respectively. Since we assume that $\Delta \ll t(s \ll u)$, it is sufficient to consider the following three cases:

$$
\begin{aligned}
& \text { (A) } \Delta \ll t \ll 1 / \lambda \quad \Longleftrightarrow \quad \lambda \ll s \ll u, \\
& \text { (B) } \Delta \ll 1 / \lambda \ll t \quad \Longleftrightarrow \quad s \ll \lambda \ll u, \\
& \text { (C) } 1 / \lambda \ll \Delta \ll t \quad \Longleftrightarrow \quad s \ll u \ll \lambda .
\end{aligned}
$$

Here it is expected that the standard random walk behavior arises in case (C). Thus, we only study the cases (A) and (B) in this paper.

\section{A. Decomposition of ensemble average of TAMSD}

In this subsection, we rewrite the ensemble averages of TAMSD $\left\langle\overline{(\delta x)^{2}}(\Delta, t)\right\rangle$ and $\left.\overline{\left\langle(\delta x)^{2}\right.}(\Delta, t)\right\rangle_{\text {eq }}$ by using the EAMSD $\left\langle|x(\Delta)-x(0)|^{2}\right\rangle$ [see Eqs. (15) and (21) ]. Here and in the followings, we use the bracket $\langle\cdot\rangle$ for the average over the non-equilibrium initial ensemble, while $\langle\cdot\rangle_{\text {eq }}$ for the average over the equilibrium initial ensemble. Also, we assume that initial position $x(0)$ is uniformly distributed on the lattice $\{1, \ldots, L\}$ for both ensembles.

First, let $w(\tau)$ be the waiting time distribution of a renewal process with a finite mean, $\langle\tau\rangle\langle\infty$. Moreover, we define $w_{e}\left(\tau ; t^{\prime}\right)$ as the PDF of the forward recurrence time $\tau$ [47], i.e., $w_{e}\left(\tau ; t^{\prime}\right)$ is the waiting time distribution at time $t^{\prime}$. Here, note that $t^{\prime}$ is not necessarily a renewal time. Particularly, $w_{\mathrm{e}}(\tau ; 0)=w(\tau)$ for the nonequilibrium initial ensemble, since we assume that we start measurements at $t^{\prime}=0$, whereas $w_{\mathrm{e}}\left(\tau ; t^{\prime}\right) \neq w(\tau)$ in general. By contrast, for the equilibrium ensemble,

$$
w_{\mathrm{e}}^{\mathrm{eq}}\left(\tau ; t^{\prime}\right)=w^{\mathrm{eq}}(\tau)
$$

where $w_{\mathrm{e}}^{\mathrm{eq}}\left(\tau ; t^{\prime}\right)$ is the PDF of the forward recurrence time at time $t^{\prime}$ for the equilibrium ensemble, and $w^{\mathrm{eq}}(\tau)$ is defined through its Laplace transformation $\tilde{w}^{\text {eq }}(s) \equiv$ $\{1-\tilde{w}(s)\} /\langle\tau\rangle s$ [See Eq. (6)]. This relation [Eq. (9)] is obvious because of the time-translation invariance of the equilibrium state, and a proof is given in Appendix C.

\section{Non-equilibrium ensemble}

Let us begin with the non-equilibrium ensemble. Using the PDF $w_{\mathrm{e}}\left(\tau ; t^{\prime}\right)$, we can express the ensemble average 
of the displacement during $\left[t^{\prime}, t^{\prime}+\Delta\right]$ as follows:

$$
\begin{aligned}
& \left\langle\left[x\left(t^{\prime}+\Delta\right)-x\left(t^{\prime}\right)\right]^{2}\right\rangle \\
& =\sum_{l= \pm 1} \frac{1}{2} \int_{0}^{\Delta} d \tau w_{\mathrm{e}}\left(\tau ; t^{\prime}\right)\left\langle\left|x\left(t^{\prime}+\Delta\right)-x\left(t^{\prime}+\tau\right)+l\right|^{2}\right\rangle \\
& =\int_{0}^{\Delta} d \tau w_{\mathrm{e}}\left(\tau ; t^{\prime}\right)\left[\left\langle|x(\Delta-\tau)-x(0)|^{2}\right\rangle+1\right]
\end{aligned}
$$

This is an exact relation, while an approximated version of this equation is already presented in Ref.[38]. We also show a more detailed derivation in Appendix D. From Eqs. (1) and (10), we have

$$
\begin{aligned}
& \left\langle\overline{(\delta x)^{2}}(\Delta, t)\right\rangle \\
& =\int_{0}^{\Delta} d \tau \bar{w}_{\mathrm{e}}(\tau ; t)\left[\left\langle|x(\Delta-\tau)-x(0)|^{2}\right\rangle+1\right],
\end{aligned}
$$

where $\bar{w}_{\mathrm{e}}(\tau ; t)$ is defined as

$$
\bar{w}_{\mathrm{e}}(\tau ; t) \equiv \int_{0}^{t-\Delta} d t^{\prime} \frac{w_{\mathrm{e}}\left(\tau ; t^{\prime}\right)}{t-\Delta} .
$$

From Eq. (11), the ensemble average of TAMSD is given by the convolution of $\bar{w}_{\mathrm{e}}(\tau ; t)$ and the EAMSD \langle|$x(\Delta-$ $\left.\tau)-\left.x(0)\right|^{2}\right\rangle$. In this and the following subsections, we study these two factors using Laplace transformations. Here we further rewrite Eqs. (11) and (12) using Laplace transformations. First, the Laplace transform of Eq. (12) with respect to $\tau$ gives

$$
\tilde{\bar{w}}_{\mathrm{e}}(u ; t)=\int_{0}^{t-\Delta} d t^{\prime} \frac{\tilde{w}_{\mathrm{e}}\left(u ; t^{\prime}\right)}{t-\Delta},
$$

where we have defined the Laplace transformations of $w_{\mathrm{e}}(\tau ; t)$ and $\bar{w}_{\mathrm{e}}(\tau ; t)$ as $\tilde{w}_{\mathrm{e}}(u ; t) \equiv \int_{0}^{\infty} w_{\mathrm{e}}(\tau ; t) e^{-u \tau} d \tau$ and $\tilde{\bar{w}}_{\mathrm{e}}(u ; t) \equiv \int_{0}^{\infty} \bar{w}_{\mathrm{e}}(\tau ; t) e^{-u \tau} d \tau$, respectively. Furthermore, the Laplace transform of Eq. (13) with respect to $t$ gives

$$
\breve{\breve{w}}_{\mathrm{e}}(u ; s)=e^{-s \Delta} \int_{s}^{\infty} d s^{\prime} \frac{\breve{w}_{\mathrm{e}}\left(u ; s^{\prime}\right)}{s^{\prime}},
$$

where the double Laplace transformations $\breve{w}_{\mathrm{e}}(u ; s)$ and $\breve{\bar{w}}_{\mathrm{e}}(u ; s)$ are defined as $\breve{w}_{\mathrm{e}}(u ; s) \equiv \int_{0}^{\infty} \tilde{w}_{\mathrm{e}}(u ; t) e^{-s t} d t$ and $\breve{\bar{w}}_{\mathrm{e}}(u ; s) \equiv \int_{\Delta}^{\infty} \tilde{\bar{w}}_{\mathrm{e}}(u ; t) e^{-s t} d t$, respectively. In addition, by taking the Laplace transformation of Eq. (11) with respect to $\Delta$, we obtain

$$
\begin{aligned}
\mathcal{L} & {\left[\left\langle\overline{(\delta x)^{2}}(\Delta, t)\right\rangle\right](u, t) } \\
& =\tilde{\bar{w}}_{\mathrm{e}}(u ; t)\left\{\mathcal{L}\left[\left\langle|x(\Delta)-x(0)|^{2}\right\rangle\right](u)+\frac{1}{u}\right\} .
\end{aligned}
$$

For the non-equilibrium ensemble, the following relation between the waiting time distribution $w(t)$ and forward recurrence time distribution $w_{\mathrm{e}}\left(\tau ; t^{\prime}\right)$ is well known [46-48]:

$$
\breve{w}_{\mathrm{e}}(u ; s)=\frac{\tilde{w}(u)-\tilde{w}(s)}{s-u} \frac{1}{1-\tilde{w}(s)} .
$$

See Appendix $\mathrm{C}$ for a derivation. If we choose TSD [Eq. (4)] for the waiting time distribution $w(\tau)$, we have

$$
\breve{w}_{\mathrm{e}}(u ; s) \simeq \frac{(\lambda+u)^{\alpha}-(\lambda+s)^{\alpha}}{u-s} \frac{1}{(\lambda+s)^{\alpha}-\lambda^{\alpha}},
$$

where we have used Eq. (2) and $\lambda, u, s \ll 1$. Using the Eq. (17) and $s \ll u$, the integral on the RHS of Eq. (14) can be approximated as

$$
\int_{s}^{\infty} d s^{\prime} \frac{\breve{w}_{\mathrm{e}}\left(u ; s^{\prime}\right)}{s^{\prime}} \simeq \begin{cases}\frac{u^{\alpha-1}}{\alpha s^{\alpha}}, & \text { for } \lambda \ll s \\ \frac{\lambda^{1-\alpha} u^{\alpha-1}}{\alpha s}, & \text { for } s \ll \lambda\end{cases}
$$

Then, the inverse Laplace transformation of Eq. (14) with respect to $s$ gives

$$
\tilde{\bar{w}}_{\mathrm{e}}(u ; t) \simeq \begin{cases}\frac{u^{\alpha-1}}{\Gamma(\alpha+1)} t^{\alpha-1}, & \text { for } t \ll 1 / \lambda \\ \frac{\lambda^{1-\alpha} u^{\alpha-1}}{\alpha}, & \text { for } 1 / \lambda \ll t\end{cases}
$$

where we have used $e^{-s \Delta} \simeq 1[$ Note that $s \Delta \ll 1$ because of Eq. (8)].

\section{Equilibrium ensemble}

For the case of the equilibrium ensemble, Eq. (10) should be replaced by

$$
\begin{aligned}
& \left\langle\left[x\left(t^{\prime}+\Delta\right)-x\left(t^{\prime}\right)\right]^{2}\right\rangle_{\mathrm{eq}} \\
& \quad=\int_{0}^{\Delta} d \tau w^{\mathrm{eq}}(\tau)\left[\left\langle|x(\Delta-\tau)-x(0)|^{2}\right\rangle+1\right] .
\end{aligned}
$$

Note that the ensemble average in the right-hand side (RHS) is taken over the non-equilibrium ensemble. Since RHS of the above equation is independent of $t^{\prime}$, we have the following equation through the calculation similar to that in the non-equilibrium case:

$$
\begin{aligned}
& \mathcal{L}\left[\left\langle\overline{(\delta x)^{2}}(\Delta)\right\rangle_{\text {eq }}\right](u) \\
& \quad=\tilde{w}^{\text {eq }}(u)\left\{\mathcal{L}\left[\left\langle|x(\Delta)-x(0)|^{2}\right\rangle\right](u)+\frac{1}{u}\right\} .
\end{aligned}
$$

Moreover, the left-hand-side (LHS) of Eq. (20) with $t^{\prime}=$ 0 is just the EAMSD with respect to the equilibrium initial ensemble. Thus, using Eq. (1) we have

$$
\left\langle[x(\Delta)-x(0)]^{2}\right\rangle_{\mathrm{eq}}=\left\langle\overline{(\delta x)^{2}}(\Delta)\right\rangle_{\mathrm{eq}}
$$

This is a manifestation of ergodicity for the equilibrium ensemble. Note that Eq. (22) is valid in general, if $\langle\tau\rangle$ is finite (and thus $w^{\mathrm{eq}}(\tau)$ exists). 
If we choose TSD [Eqs. (4) and (2)] for the waiting time distribution $w(\tau)$, we have the following relation for $\tilde{w}^{\mathrm{eq}}(u)[47]$ :

$$
\tilde{w}^{\mathrm{eq}}(u)=\frac{1-e^{-c\left[(\lambda+u)^{\alpha}-\lambda^{\alpha}\right]}}{\langle\tau\rangle u} \simeq \frac{1}{\alpha}\left(\frac{\lambda}{u}\right)^{1-\alpha},
$$

where we used the assumption $\lambda \ll u$ [Eq. (8)] and $\langle\tau\rangle=$ $c \lambda^{\alpha-1} \alpha$.

\section{B. Ensemble average of MSD}

Furthermore, we have to calculate the Laplace transform of EAMSD $\left\langle|x(\Delta)-x(0)|^{2}\right\rangle$ in order to obtain the ensemble average of TAMSD [Eqs. (15) and (21)]. First, let us express the EAMSD approximately as follows [38]:

$\left\langle|x(\Delta)-x(0)|^{2}\right\rangle \simeq \int_{0}^{L} \frac{d x_{s}}{L} \int_{0}^{L} d x P\left(x, \Delta ; x_{s}, 0\right)\left(x-x_{s}\right)^{2}$,

where $P\left(x, \tau ; x_{s}, 0\right)$ is the transition probability from $x_{s}(t=0)$ to $x(t=\Delta)$ of the GFFPE (See Appendix $\mathrm{F})$. The above equation is correct in the hydrodynamic limit $L \rightarrow \infty$. Also, it should be noted that the GFFPE is an equation for the non-equilibrium ensemble, and therefore the EAMSD given by Eq. (24) is also for the non-equilibrium ensemble. The Laplace transformation of Eq. (24) is given by

$$
\begin{aligned}
\mathcal{L} & {\left[\left\langle|x(\Delta)-x(0)|^{2}\right\rangle\right](u) } \\
& =\int_{0}^{L} \frac{d x_{s}}{L} \int_{0}^{L} d x \tilde{P}\left(x, u ; x_{s}, 0\right)\left(x-x_{s}\right)^{2} \\
& =\frac{L^{2}}{6}\left[\frac{1}{u}-\frac{96}{\pi^{4}} \sum_{\substack{n=1 \\
n \text { :odd }}}^{\infty} \frac{n^{-4}}{u+(n \pi / L)^{2} K u \tilde{M}(u)}\right] \\
& \simeq \frac{L^{2}}{6 u}\left[1-\frac{96}{\pi^{4}} \sum_{\substack{n=1 \\
n: \text { odd }}}^{\infty} \frac{1}{n^{4}} \frac{\left(\Delta_{c} u\right)^{\alpha}}{\left(\Delta_{c} u\right)^{\alpha}+n^{2}}\right]
\end{aligned}
$$

where we use $\lambda \ll u(\Delta \ll 1 / \lambda)$. Moreover, $K$ is defined as $K \equiv 1 / 2 c$, and ' $n$ : odd' under the $\sum$ means that the summation is taken over odd terms. We also define a characteristic timescale $\Delta_{c}$ as

$$
\Delta_{c} \equiv\left(\frac{2 c L^{2}}{\pi^{2}}\right)^{1 / \alpha}
$$

Note that Eq. (25) is the same as the one obtained in [38] for the case of the power-law waiting time. This means that this property of EAMSD is independent of the timescale of the smooth cutoff $1 / \lambda$.

Finally, we obtain the following estimate for the Laplace transformation of EAMSD:

$$
\mathcal{L}\left[\left\langle|x(\Delta)-x(0)|^{2}\right\rangle\right](u) \simeq \begin{cases}\frac{1}{c u^{1+\alpha}}, & \text { for } \Delta_{c} u \gg 1 \\ \frac{L^{2}}{6 u}, & \text { for } \Delta_{c} u \ll 1,\end{cases}
$$

where we used the RHS of Eq. (25) for the case of $\Delta_{c} u \ll$ 1, while we rewrote the RHS of Eq. (25) by using zeta functions (see Appendix G) for the case of $\Delta_{c} u \gg 1$ as

$$
\frac{16 L^{2}}{\pi^{4} \Delta_{c}^{\alpha} u^{1+\alpha}}\left[\frac{\pi^{2}}{8}-\sum_{\substack{n=1 \\ n \text { :odd }}}^{\infty} \frac{1}{\left(\Delta_{c} u\right)^{\alpha}+n^{2}}\right] .
$$

Then, the summation term can be neglected since $\Delta_{c} u \gg$ 1. From Eq. (27), we obtain the EAMSD for nonequilibrium ensemble as follows:

$$
\left\langle|x(\Delta)-x(0)|^{2}\right\rangle \simeq \begin{cases}\frac{\Delta^{\alpha}}{c \Gamma(1+\alpha)}, & \text { for } \Delta \ll \Delta_{c} \\ \frac{L^{2}}{6}, & \text { for } \Delta \gg \Delta_{c} .\end{cases}
$$

As shown in the next subsection, the TAMSD for the non-equilibrium ensemble behaves differently. Namely, the ergodicity is broken even at $t \rightarrow \infty$. In contrast, the ergodicity is satisfied for the equilibrium ensemble at $t \rightarrow \infty$.

\section{Ensemble average of TAMSD}

In this subsection, we derive asymptotic behavior of the ensemble-averaged TAMSD using the results from the preceding subsections.

\section{Non-equilibrium ensemble [case $(A)]$ : $t \ll 1 / \lambda$}

First, we start with the non-equilibrium ensemble for $t \ll 1 / \lambda$. From Eqs. (15), (19), and (27), we have leading terms in $u \ll 1$ and $L \gg 1$ as follows:

$$
\begin{aligned}
& \left.\mathcal{L}\left[\overline{\left\langle(\delta x)^{2}\right.}(\Delta, t)\right\rangle\right](u, t) \\
& = \begin{cases}\frac{u^{-2}}{c \Gamma(1+\alpha) t^{1-\alpha}}, & \text { for } \Delta_{c} u \gg 1 \\
\frac{L^{2}}{6} \frac{u^{\alpha-2}}{\Gamma(1+\alpha) t^{1-\alpha}}, & \text { for } \Delta_{c} u \ll 1 .\end{cases}
\end{aligned}
$$

The inverse Laplace transformation with respect to $u$ gives

$$
\left\langle\overline{(\delta x)^{2}}(\Delta, t)\right\rangle=\left\{\begin{array}{lr}
\frac{\Delta}{c \Gamma(1+\alpha) t^{1-\alpha}}, & \text { for } \Delta \ll \Delta_{c} \\
\frac{L^{2} \Delta^{1-\alpha}}{6 \Gamma(1+\alpha) \Gamma(2-\alpha) t^{1-\alpha}}, & \text { for } \Delta_{c} \ll \Delta .
\end{array}\right.
$$



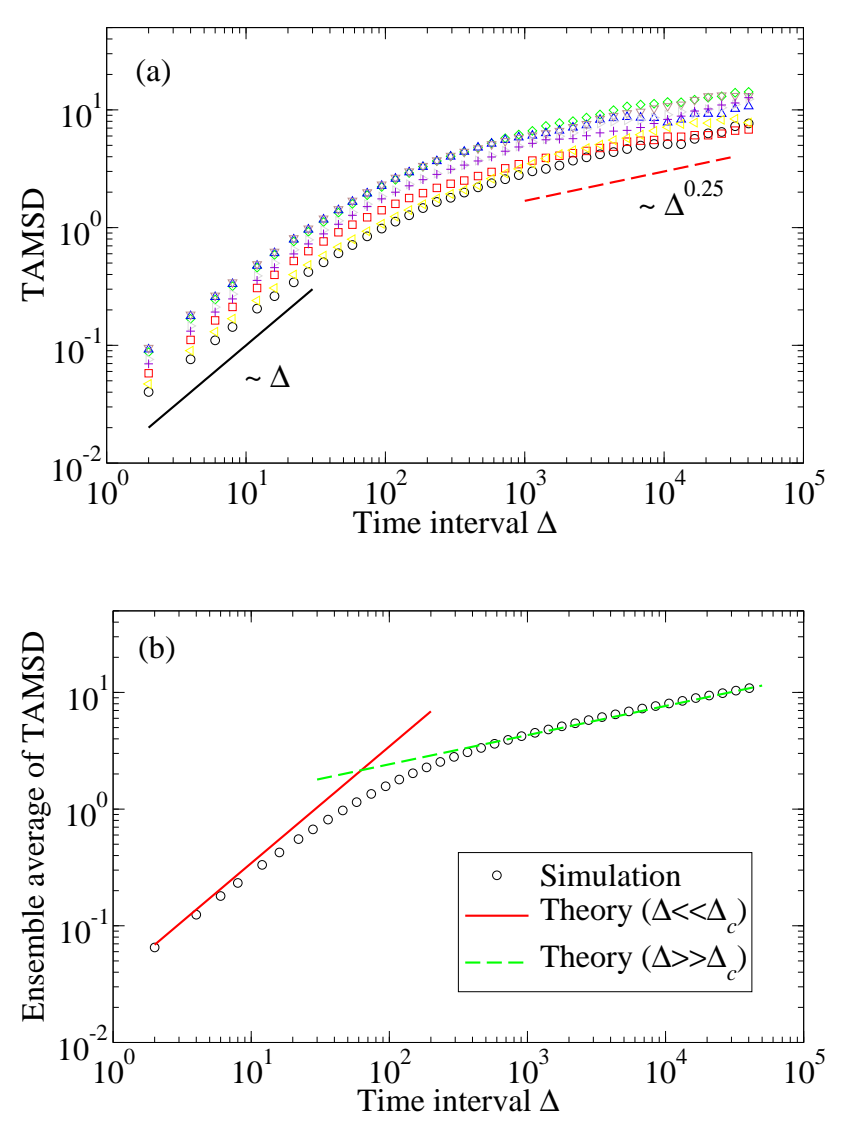

FIG. 1. (Color online) (a) TAMSD $\overline{(\delta x)^{2}}(\Delta, t)$ vs. time interval $\Delta$ in $\log -\log$ form for the non-equilibrium ensemble $(\mathrm{A})$ : $t<1 / \lambda$. The total measurement time $t$ is set as $t=10^{6}$, and the cutoff parameter $\lambda$ as $\lambda=10^{-7}$. Other parameters are set as $\alpha=0.75, c=1$, and $L=11$. TAMSD is calculated for 8 different realizations of trajectories and different symbols correspond to different realizations. (b) The ensemble average of TAMSD in $\log -\log$ form (circles). The lines are the theoretical predictions given by Eq. (31). Note that no adjustable parameters were used to obtain these theoretical lines.

Thus, the ensemble-averaged TAMSD shows normal diffusion at a short timescale $\left(\Delta \ll \Delta_{c}\right)$ and anomalous slow diffusion at a longer timescale $\left(\Delta_{c} \ll \Delta\right.$.). As expected, these results perfectly coincide with those of the previous studies [Eqs. (10) and (11) in [38]]. Also, in this regime, the TAMSD depends on the measurement time $t$. That is, the diffusion becomes slower, as the measurement time increases. We call this behavior aging in this article.

In Fig. 1(a), TAMSDs for 8 different trajectories are shown. Although these TAMSDs show similar scaling behavior, the diffusion constant $D$ of each TAMSD $\overline{(\delta x)^{2}}(\Delta, t) \sim D \Delta^{\gamma}$ seems randomly distributed. This behavior is analyzed in the next section. In Fig. 1 (b), the ensemble-averaged TAMSD $\left\langle\overline{(\delta x)^{2}}(\Delta, t)\right\rangle$ is displayed. The solid and dashed lines are the theoretical predictions given by Eq. (31).
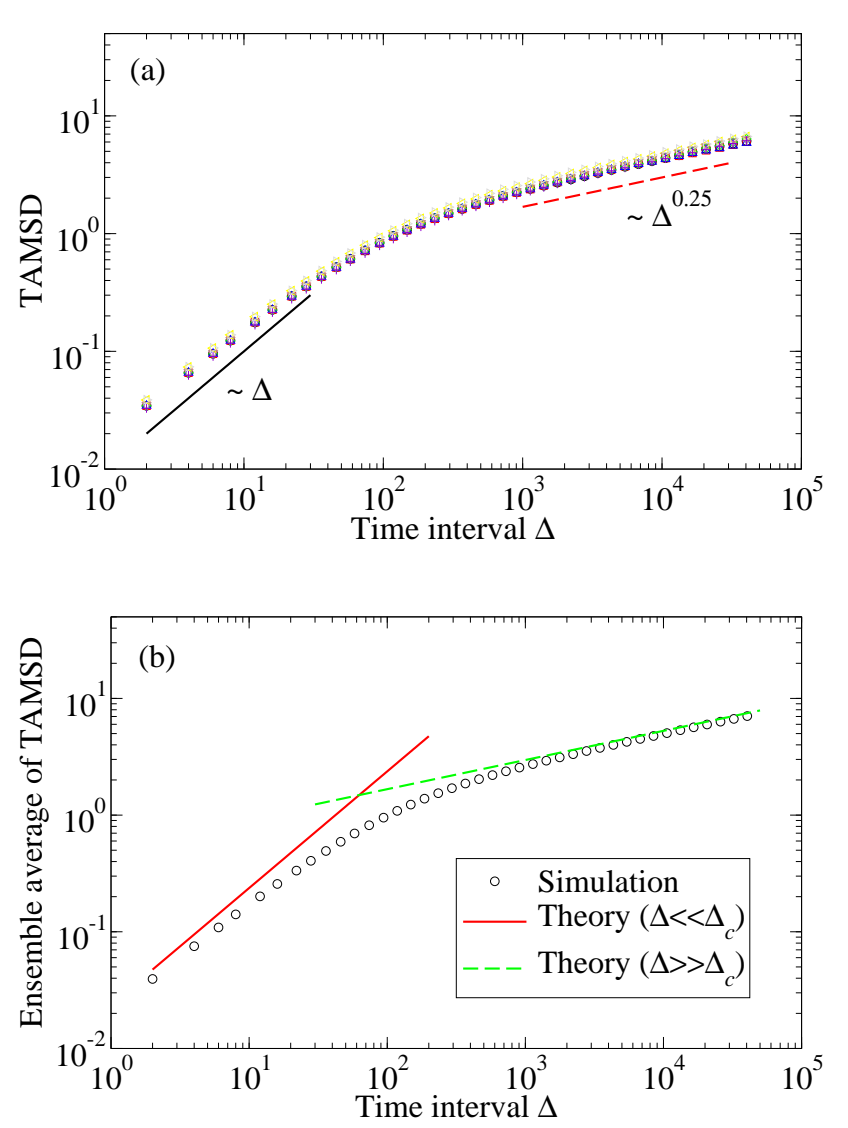

FIG. 2. (Color online) (a) TAMSD $\overline{(\delta x)^{2}}(\Delta, t)$ vs. time interval $\Delta$ in $\log -\log$ form for the non-equilibrium ensemble (B): $t>1 / \lambda$. The total measurement time $t$ is set as $t=10^{8}$, and the cutoff parameter $\lambda$ as $\lambda=10^{-7}$. The other parameters are the same as in Fig. 1. TAMSD is calculated for 8 different realizations of trajectories and different symbols correspond to different realizations. (b) The ensemble average of TAMSD in $\log -\log$ form (circles). The lines are theoretical predictions given by Eq. (33). No adjustable parameters were used to obtain these theoretical lines.

\section{Non-equilibrium ensemble [case $(B)]: 1 / \lambda \ll t$}

Next, we study the case of $1 / \lambda \ll t$. From Eqs. (15), (19), and (27), we have

$$
\mathcal{L}\left[\left\langle\overline{(\delta x)^{2}}(\Delta, t)\right\rangle\right](u, t)= \begin{cases}\frac{1}{c \lambda^{\alpha-1} \alpha} \frac{1}{u^{2}}, & \text { for } \Delta_{c} u \gg 1 \\ \frac{1}{\lambda^{\alpha-1} \alpha} \frac{L^{2}}{6 u^{2-\alpha}}, & \text { for } \Delta_{c} u \ll 1 .\end{cases}
$$

Then, taking the inverse Laplace transformation, we obtain

$$
\left\langle\overline{(\delta x)^{2}}(\Delta, t)\right\rangle= \begin{cases}\frac{\Delta}{c \lambda^{\alpha-1} \alpha}, & \text { for } \Delta \ll \Delta_{c} \\ \frac{1}{\lambda^{\alpha-1} \alpha} \frac{L^{2} \Delta^{1-\alpha}}{6 \Gamma(2-\alpha)}, & \text { for } \Delta_{c} \ll \Delta .\end{cases}
$$

Thus, a crossover from the normal to anomalous diffusion similar to that in case (A) can be observed even 
in the long measurement time limit $t \rightarrow \infty$, whereas the aging behavior - $t$-dependence of the diffusion constantvanishes. Note also that even in this limit, $t \rightarrow \infty$, the (ensemble-averaged) TAMSD does not coincide with the EAMSD [Eq. (29)].

Fig. 2(a) shows TAMSDs for 8 different trajectories. In this case, the scatter of TAMSDs, observed in Fig. 1(a), is diminished. The ensemble-averaged TAMSD is also shown in Fig. 2(b) by circles, where the theoretical predictions given by Eq. (33) are shown by solid and dashed lines.

\section{Equilibrium ensemble}

For the case of the equilibrium initial ensemble, we have the following relation from Eqs. (21), (23), and (27):

$$
\mathcal{L}\left[\left\langle\overline{(\delta x)^{2}}(\Delta)\right\rangle_{\text {eq }}\right](u) \simeq \begin{cases}\frac{1}{c \lambda^{\alpha-1} \alpha} \frac{1}{u^{2}}, & \text { for } \Delta_{c} u \gg 1 \\ \frac{1}{\lambda^{\alpha-1} \alpha} \frac{L^{2}}{6 u^{2-\alpha}}, & \text { for } \Delta_{c} u \ll 1 .\end{cases}
$$

Through the inverse transformation, we have

$$
\left.\overline{\left\langle(\delta x)^{2}\right.}(\Delta)\right\rangle_{\mathrm{eq}} \simeq \begin{cases}\frac{\Delta}{c \lambda^{\alpha-1} \alpha}, & \text { for } \Delta \ll \Delta_{c} \\ \frac{1}{\lambda^{\alpha-1} \alpha} \frac{L^{2} \Delta^{1-\alpha}}{6 \Gamma(2-\alpha)}, & \text { for } \Delta \gg \Delta_{c} .\end{cases}
$$

The equation (35) is equivalent to Eq. (33), the TAMSD for the non-equilibrium ensemble [case (B)]. However, Eq. (35) is valid for arbitrary measurement times $t$, while Eq. (33) is valid only for long measurement times $(1 / \lambda \ll t)$. In addition, the aging behavior is absent in the equilibrium case.

In Fig. 3(a) and (b), TAMSDs for 8 different trajectories are shown for a short and long measurement times $t$, respectively. Surprisingly, the scatter of TAMSD is even broader than that in the non-equilibrium case [Fig. 1(a)] at short measurement times $t$ as shown in Fig. 3(a).

In summary, for the non-equilibrium ensemble, the scatter of TAMSD appears with the aging behavior [the non-equilibrium ensemble (case A)], whereas for the equilibrium ensemble the scatter appears without aging. These scatters are also studied quantitatively through theoretical analysis in the next section. The theoretical predictions given by Eq. (35) are also shown by dotted and dashed lines in Fig. 3(b).

Furthermore, from Eq. (22), the EAMSD for the equilibrium initial ensemble $\left\langle[x(\Delta)-x(0)]^{2}\right\rangle_{\text {eq }}$ is also given by the RHS of Eq. (35). In Fig. 3(b), a numerically obtained EAMSD $\left\langle[x(\Delta)-x(0)]^{2}\right\rangle_{\text {eq }}$ is displayed by a
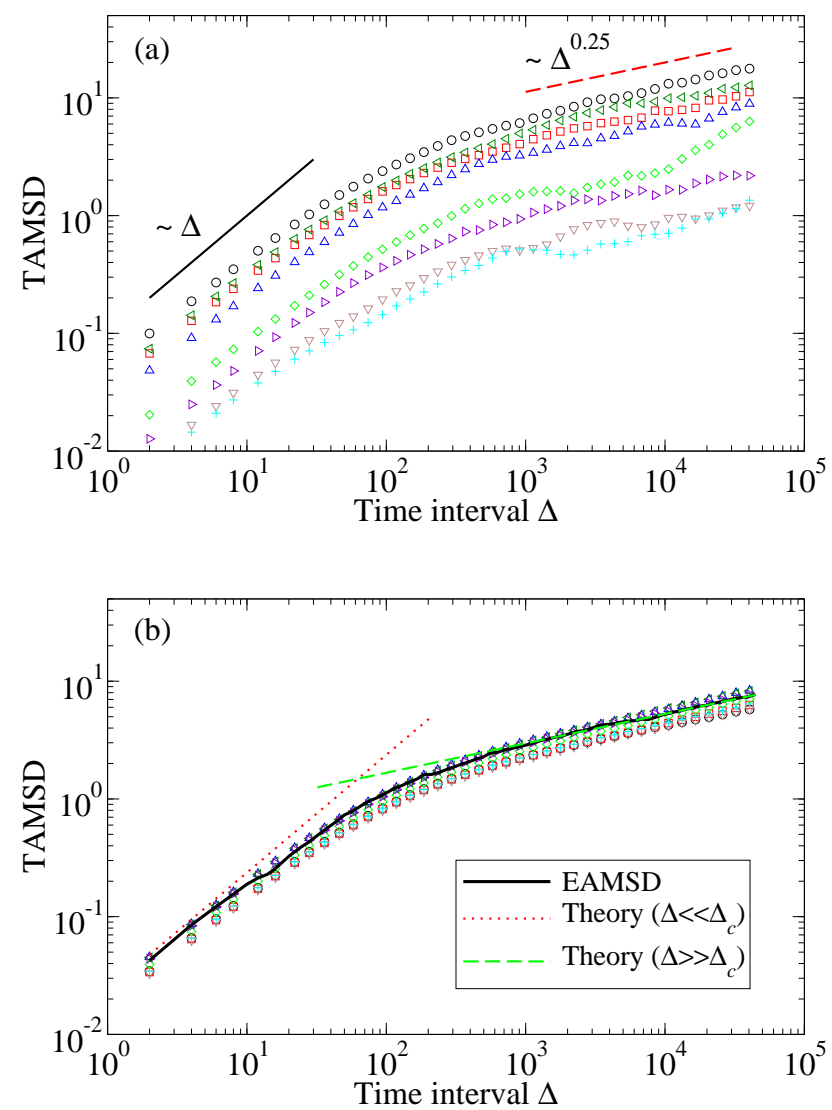

FIG. 3. (Color online) (a) TAMSD $\overline{(\delta x)^{2}}(\Delta, t)$ vs. time interval $\Delta$ in $\log -\log$ form for the equilibrium ensemble with $t<1 / \lambda$. The total measurement time $t$ is set as $t=10^{6}$, and the cutoff parameter $\lambda$ as $\lambda=10^{-7}$. The other parameters are the same as in Fig. 1. TAMSD is calculated for 8 different realizations of trajectories and different symbols correspond to different realizations. (b) The same as the figure (a) except that $t=10^{8}(t>1 / \lambda)$. The symbols are TAMSDs for 8 different trajectories and the thick solid curve is the EAMSD $\left\langle[x(\Delta)-x(0)]^{2}\right\rangle_{\text {eq }}$. The dotted and dashed lines are theoretical predictions given by Eq. (35). Note that no adjustable parameters were used to obtain these theoretical lines.

solid curve, which is consistent with the theory [Eq. (35): the dotted and dashed lines in Fig. 3(b)]. Thus, the EAMSD for the equilibrium ensemble $\left\langle[x(\Delta)-x(0)]^{2}\right\rangle_{\text {eq }}$ [Eq. (35)] is different from the EAMSD for the nonequilibrium ensemble $\left\langle[x(\Delta)-x(0)]^{2}\right\rangle$ [Eq. (29)].

\section{STATISTICAL AND ERGODIC PROPERTIES OF TAMSD}

The statistical property of TAMSD is dominated by the property of the number of jumps $N_{t}$ until time $t$ (see Sec. IV C). Therefore, we first study $N_{t}$ in Secs. IV A and IV B on the basis of the analysis presented in [23]. 


\section{A. Real space analysis}

In the Sec. II, we define $t_{n}$ as the time when the $n$-th jump occurs for a trajectory $x(t): t_{n}=\sum_{k=1}^{n} \tau_{k}$. From this definition, we have the following relation:

$$
\begin{aligned}
G(n ; t) & \equiv \operatorname{Prob}\left(N_{t}<n\right) \\
& =\operatorname{Prob}\left(t_{n}>t\right)=\operatorname{Prob}\left(\sum_{k=1}^{n} \tau_{k}>t\right),
\end{aligned}
$$

where $\operatorname{Prob}(\ldots)$ is the probability and $\tau_{k}(k=1,2, \ldots)$ are the waiting times between jumps.

\section{Non-equilibrium ensemble}

From Eq. (36), we have the following equation for the non-equilibrium ensemble:

$$
\begin{aligned}
\operatorname{Prob}\left(N_{t}<n\right) & =\int_{t}^{\infty} d \tau P_{\mathrm{TL}}^{n}(\tau, \lambda) \\
& =\int_{n^{-1 / \alpha} t}^{\infty} d \tau P_{\mathrm{TL}}\left(\tau, n^{1 / \alpha} \lambda\right),
\end{aligned}
$$

where we used the statistical independence between the waiting times $\tau_{k}(k=1,2, \ldots)$ and Eq. (5). Furthermore, if we change variables from $n$ to $x$ as $n=t^{\alpha} x$, we obtain

$$
\begin{aligned}
\operatorname{Prob}\left(\frac{N_{t}}{t^{\alpha}}<x\right) & =\int_{x^{-1 / \alpha}}^{\infty} d \tau P_{\mathrm{TL}}\left(\tau, t x^{1 / \alpha} \lambda\right) \\
& =\int_{0}^{x} \frac{d \tau}{\alpha \tau^{1+1 / \alpha}} P_{\mathrm{TL}}\left(\tau^{-1 / \alpha}, t x^{1 / \alpha} \lambda\right) .
\end{aligned}
$$

Note that the integrand of the RHS of Eq. (38) is not the PDF, because it contains the variable $x$. To derive the PDF for $x$, we insert Eq. (4) into Eq. (38), and then we have

$$
\begin{aligned}
\operatorname{Prob}\left(\frac{N_{t}}{t^{\alpha}}<x\right)= & -\frac{e^{c(t \lambda)^{\alpha} x}}{\alpha \pi} \sum_{k=1}^{\infty} \frac{\Gamma(k \alpha+1)}{k ! k} \\
& \times(-c x)^{k} \sin (\pi k \alpha) a_{k},
\end{aligned}
$$

where

$$
a_{k} \equiv \int_{0}^{1} d \tau e^{-t \lambda \tau^{-1 /(\alpha k)}}
$$

Differentiating Eq. (39) in terms of $x$, we obtain the PDF for $x$ :

$$
\begin{aligned}
f_{\lambda}(x ; t)= & -\frac{e^{c(t \lambda)^{\alpha} x}}{\alpha \pi} \sum_{k=1}^{\infty} \frac{\Gamma(k \alpha+1)}{k !}(-c)^{k} \\
& \times\left[\frac{c(t \lambda)^{\alpha} x}{k}+1\right] x^{k-1} \sin (\pi k \alpha) a_{k} .
\end{aligned}
$$

When $\lambda=0$, this PDF $f_{0}(x) \equiv f_{0}(x, t)$ is called the Mittag-Leffler distribution [28, 29].
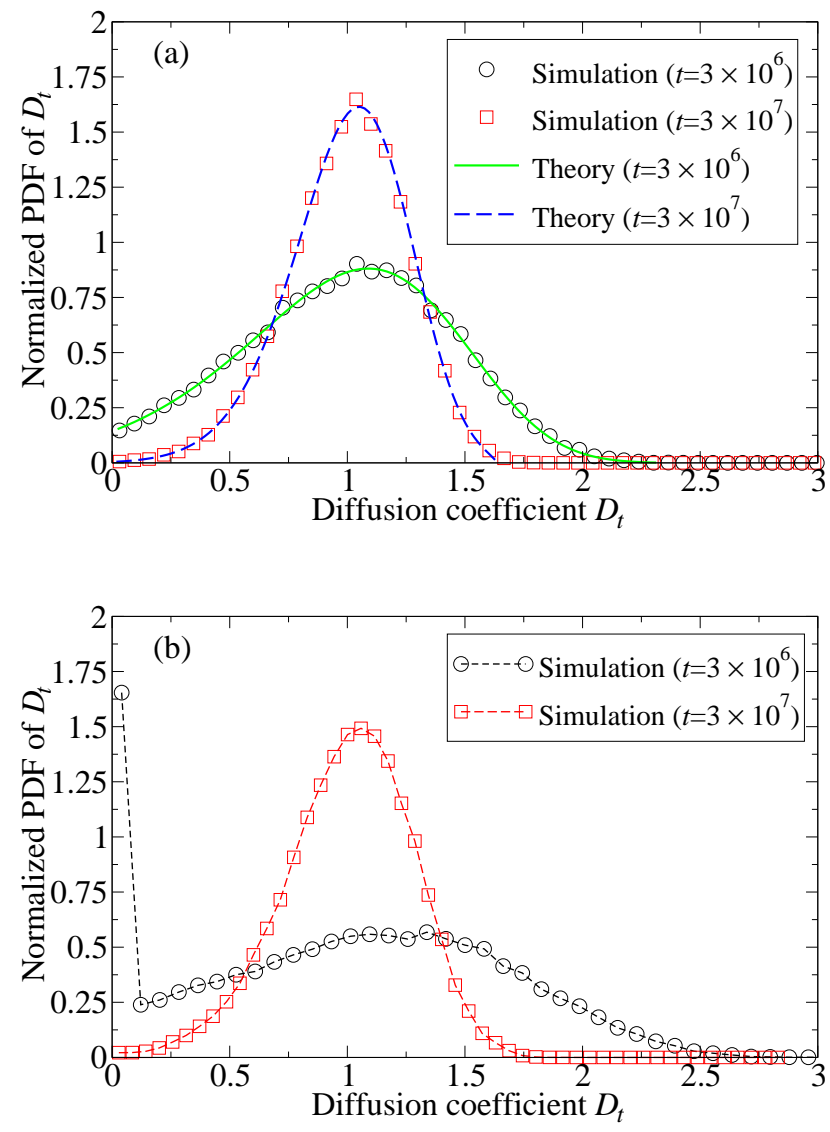

FIG. 4. (color online) (a) PDF of the diffusion coefficient $D_{t}$ for the non-equilibrium TAMSD $\overline{(\delta x)^{2}}(\Delta, t) \approx D_{t} \Delta$ for small $\Delta$. Each PDF is normalized so that its mean value equals unity. $D_{t}$ is calculated from TAMSD by least-square fitting over the interval $0<\Delta<10$. The results for two different values of measurement times are presented: $t=3 \times 10^{6}$ (circles) and $3 \times 10^{7}$ (squares). The other parameters are set as $\lambda=10^{-7}, \alpha=0.75, c=1$, and $L=11$. The lines correspond to the theoretical predictions given by Eq. (41). No adjustable parameters were used to obtain these curves. (b) PDF of the diffusion coefficient $D_{t}$ for the equilibrium TAMSD $\overline{(\delta x)^{2}}(\Delta, t) \approx D_{t} \Delta$ for small $\Delta$. The parameter values are the same as in the figure (a). The lines are to guide the eye.

As shown below [Sec. IV C], TAMSD behaves as $\overline{(\delta x)^{2}}(\Delta, t) \approx \frac{N_{t}}{t} \Delta$ for small $\Delta$. Thus, the diffusion constant $D_{t}$, defined as $\overline{(\delta x)^{2}}(\Delta, t) \approx D_{t} \Delta$, shows the same statistical property as that of $N_{t}$. Fig. 4 shows the PDF of $D_{t}$ for two different measurement times $t$. Note that the PDF narrows as $t$ increases. The analytical results given in Eq. (41) are also depicted by solid and dashed lines in the figure. 


\section{Equilibrium ensemble}

The equilibrium case is also analyzed in a similar way. In this case, Eq. (37) should be replaced by

$$
\operatorname{Prob}\left(N_{t}<n\right)=\int_{t}^{\infty} d \tau\left(P_{\mathrm{TL}}^{\mathrm{eq}} * P_{\mathrm{TL}}^{n-1}\right)(\tau, \lambda)
$$

where $(f * g)$ means a convolution. In contrast to the nonequilibrium case, however, it seems difficult to obtain a simple expression of the PDF of $N_{t}$ for the equilibrium case. As shown in Fig. 4, a qualitative difference appears for short measurement time regime, $t<1 / \lambda$. In fact, there is a peak at $D_{t}=0$ for the equilibrium initial ensemble, which is due to the mean waiting time of $P_{\mathrm{TL}}^{\mathrm{eq}}(\tau, \lambda),\langle\tau\rangle_{\mathrm{eq}}(\sim 1 / \lambda)$, is much longer than that of $P_{\mathrm{TL}}(\tau, \lambda),\langle\tau\rangle\left(\sim 1 / \lambda^{1-\alpha}\right)$. Namely, there are many trajectories that are trapped more than the measurement time $t(<1 / \lambda)[49]$.

\section{B. Laplace space analysis}

Next, to clarify the ergodic properties of the system, we study the relative standard deviation (RSD) $R(t) \equiv \sqrt{\left\langle N_{t}^{2}\right\rangle_{c}} /\left\langle N_{t}\right\rangle$, where $\langle\cdot\rangle_{c}$ is the cumulant. The quantity $R(t)$ is a measure of ergodicity [26]. In fact, if $R(t)=0$, time averages of an observable give the same value independent of the trajectory; however, if $R(t)>0$, they are different from one trajectory to another. This quantity $R(t)$ was also used in some molecular dynamics simulations to characterize the ergodicity breaking and non-Gaussian fluctuations of lipid motions in cell membranes [50], and to determine the longest relaxation time in entangled polymers [50].

\section{Non-equilibrium ensemble}

Let us begin with the non-equilibrium ensemble. In order to derive an analytical expression of $R(t)$, we start with the Laplace transformation of Eq. (37):

$$
\begin{aligned}
\tilde{G}(n ; s) & =\int_{0}^{\infty} d t e^{-t s} \int_{n^{-1 / \alpha} t}^{\infty} d \tau P_{\mathrm{TL}}\left(\tau, n^{1 / \alpha} \lambda\right) \\
& =\frac{1-e^{-n c\left[(\lambda+s)^{\alpha}-\lambda^{\alpha}\right]}}{s}
\end{aligned}
$$

where $\tilde{G}(n ; s)$ is the Laplace transformation of $G(n ; t)$ in terms of $t$. Next, let us define a function $g(n ; s)$ as $g(n ; s) \equiv \tilde{G}(n+1 ; s)-\tilde{G}(n ; s)$. Note that $g(n ; s)$ is the Laplace transformation (with respect to $t$ ) of the PDF of $N_{t}$. Furthermore, we perform a discrete Laplace transformation of $g(n ; s)$ with respect to $n$ as follows:

$$
\tilde{g}(\nu ; s)=\frac{1}{s} \frac{1-\exp \left(-c\left[(\lambda+s)^{\alpha}-\lambda^{\alpha}\right]\right)}{1-\exp \left(-\nu-c\left[(\lambda+s)^{\alpha}-\lambda^{\alpha}\right]\right)},
$$

where we define $\tilde{g}(\nu ; s)$ as $\tilde{g}(\nu ; s) \equiv \sum_{n=0}^{\infty} e^{-n \nu} g(n ; s)$. Using the assumption $s, \lambda, \nu \ll 1$, we have

$$
\tilde{g}(\nu ; s)=\frac{1}{s} \sum_{k=0}^{\infty}\left(-\frac{\nu}{c}\right)^{k}\left[(\lambda+s)^{\alpha}-\lambda^{\alpha}\right]^{-k} .
$$

a. First moments From Eq. (45), we obtain the Laplace transform $\mathcal{L}\left[\left\langle N_{t}\right\rangle\right](s)$ of the first moment $\left\langle N_{t}\right\rangle$ as

$\mathcal{L}\left[\left\langle N_{t}\right\rangle\right](s) \simeq \begin{cases}\frac{1}{c s^{\alpha+1}}, & s \gg \lambda \\ \frac{1}{c \lambda^{\alpha-1} \alpha s^{2}}\left[1+(1-\alpha) \frac{s}{2 \lambda}\right], & s \ll \lambda .\end{cases}$

The inverse Laplace transform of the above equation is given by

$$
\left\langle N_{t}\right\rangle \simeq \begin{cases}\frac{t^{\alpha}}{c \Gamma(\alpha+1)}, & t \ll 1 / \lambda \\ \frac{t}{c \lambda^{\alpha-1} \alpha}+\frac{1-\alpha}{2 c \lambda^{\alpha} \alpha}, & t \gg 1 / \lambda\end{cases}
$$

The ensemble-averaged mean square displacement (EAMSD) for free CTRWs (i.e., CTRWs without confinement effects) is known to be proportional to $\left\langle N_{t}\right\rangle$ [11]: $\left\langle(\delta \boldsymbol{r})^{2}\right\rangle(t) \sim\left\langle N_{t}\right\rangle$. Thus, the EAMSD of the present model without the effect of confinement shows transient subdiffusion, i.e., subdiffusion for short timescales and normal diffusion for long timescales [13]. The crossover time between these two regimes is characterized by $1 / \lambda$.

In addition, $\left\langle N_{t}\right\rangle$ is usually called the renewal function in the renewal theory, and Eqs. (46) and (47) can also be derived by the renewal equation [46]. In contrast, higher order moments of $N_{t}$ cannot be derived from the renewal equation, and we have to use Eq. (45).

b. Second moments Similarly, the Laplace transform $\mathcal{L}\left[\left\langle N_{t}^{2}\right\rangle\right](s)$ of the second moment $\left\langle N_{t}^{2}\right\rangle$ is given by

$\mathcal{L}\left[\left\langle N_{t}^{2}\right\rangle\right](s) \simeq \begin{cases}\frac{2}{c^{2} s^{2 \alpha+1}}, & s \gg \lambda \\ \frac{2}{c^{2} \lambda^{2 \alpha-2} \alpha^{2} s^{3}}\left[1+(1-\alpha) \frac{s}{\lambda}\right], & s \ll \lambda .\end{cases}$

Then, the inverse transform is given by

$$
\left\langle N_{t}^{2}\right\rangle \simeq \begin{cases}\frac{2 t^{2 \alpha}}{c^{2} \Gamma(2 \alpha+1)}, & t \ll 1 / \lambda \\ \frac{2}{c^{2} \lambda^{2 \alpha-2} \alpha^{2}}\left[\frac{t^{2}}{2}+\frac{1-\alpha}{\lambda} t\right], & t \gg 1 / \lambda .\end{cases}
$$

c. Relative standard deviation Using Eqs. (47) and (49), we obtain asymptotics of the RSD $\sqrt{\left\langle N_{t}^{2}\right\rangle_{c}} /\left\langle N_{t}\right\rangle$ :

$$
\frac{\sqrt{\left\langle N_{t}^{2}\right\rangle_{c}}}{\left\langle N_{t}\right\rangle} \simeq \begin{cases}\sqrt{\frac{2 \Gamma^{2}(\alpha+1)}{\Gamma(2 \alpha+1)}-1,} & t \ll 1 / \lambda \\ \sqrt{\frac{1-\alpha}{\lambda t}} & t \gg 1 / \lambda .\end{cases}
$$


We define a crossover time $t_{c}$ between the two regimes, $t \ll 1 / \lambda$ and $t \gg 1 / \lambda$, as the intersection of the two functions in Eqs. (50); therefore, we have

$$
t_{c}=\frac{(1-\alpha)}{\frac{2 \Gamma^{2}(\alpha+1)}{\Gamma(2 \alpha+1)}-1} \lambda^{-1}
$$

As shown in Fig. 5, the RSD remains almost constant before the crossover time $t_{c}$, and starts decaying rapidly after the crossover. In the figure, the RSD for the exponential waiting-time distribution, which has the same mean waiting time $\langle\tau\rangle$ as that of the TSD with $\lambda=10^{-6}$, is also shown by pluses. It is clear that the RSD for the exponential distribution (pluses) decays much more rapidly than that for the TSD (triangles).

\section{Equilibrium ensemble}

The calculation for the equilibrium case is almost parallel to that for the non-equilibrium case except that we should use Eq. (42) instead of Eq. (37). Thus, we only show the final results. First, the generating function $\tilde{g}(\nu ; s)$ is given by

$$
\tilde{g}(\nu ; s)=\frac{1}{\lambda^{\alpha-1} \alpha s^{2}} \sum_{k=0}^{\infty}\left(-\frac{\nu}{c}\right)^{k}\left[(\lambda+s)^{\alpha}-\lambda^{\alpha}\right]^{-k+1} .
$$

Using this function, we obtain $\langle N(t)\rangle_{\mathrm{eq}}$

$$
\left\langle N_{t}\right\rangle_{\mathrm{eq}} \simeq \frac{t}{c \lambda^{\alpha-1} \alpha}
$$

and the RSD for the equilibrium initial ensemble

$$
\frac{\sqrt{\left\langle N_{t}^{2}\right\rangle_{\mathrm{eq}, c}}}{\left\langle N_{t}\right\rangle_{\mathrm{eq}}} \simeq \begin{cases}\sqrt{\frac{2 \alpha}{\Gamma(2+\alpha)(\lambda t)^{1-\alpha}},} & t \ll 1 / \lambda \\ \sqrt{\frac{1-\alpha}{\lambda t}} & t \gg 1 / \lambda .\end{cases}
$$

Although the RSD slowly decays at the short measurement timescales, the relative fluctuations are even larger than the non-equilibrium case as shown in Fig. 5 .

\section{Statistical properties of TAMSD}

In this section, we discuss the statistical properties of TAMSD for one-dimensional CTRWs, although the analysis for free CTRWs in the followings can be generalized for higher dimensional systems [23]. Properties applicable to a general class of observables are summarized in Appendix H.

Here, we show that the TAMSD can be approximately
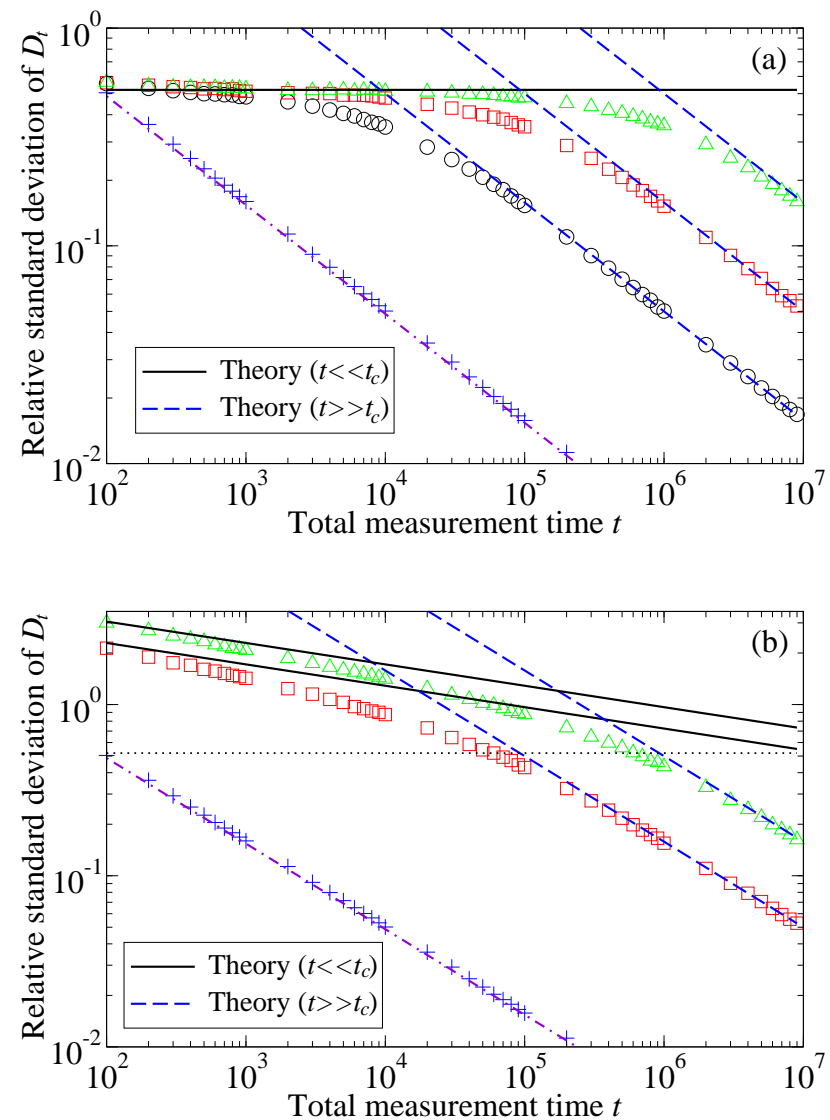

FIG. 5. (color online) (a) RSD $\sqrt{\left\langle D_{t}^{2}\right\rangle_{c}} /\left\langle D_{t}\right\rangle$ vs. total measurement time $t$ for the non-equilibrium initial ensemble. $D_{t}$ is calculated from the TAMSD $\overline{(\delta x)^{2}}(\Delta, t)$ by a least-square fitting over the interval $0<\Delta<1$. Three different values of $\lambda$ are used: $\lambda=10^{-4}$ (circles), $10^{-5}$ (squares), and $10^{-6}$ (triangles). In addition, $\alpha, c$, and $L$ are set as $\alpha=0.75$, $c=1$, and $L=11$, respectively. The lines correspond to the theoretical predictions given by Eq. (50); the solid line is the result for short timescales $t \ll t_{c}$, and the dashed lines are for long timescales $t \gg t_{c}$. The intersections of the solid and dashed lines correspond to the crossover times $t_{c}$ given by Eq. (51). (b) RSD $\sqrt{\left\langle D_{t}^{2}\right\rangle_{\text {eq }, c}} /\left\langle D_{t}\right\rangle_{\text {eq }}$ vs. total measurement time $t$ for the equilibrium initial ensemble. The parameter values are the same as those in the figure (a), except that the results only for two different values of $\lambda$ are plotted for clarity: $\lambda=10^{-5}$ (squares), and $10^{-6}$ (triangles). The lines correspond to the theoretical predictions given by Eq. (54). (a) and (b) The plus signs in both figures are the RSD for the case in which the waiting time distribution is given by the exponential distribution $P(\tau)=\exp (\tau /\langle\tau\rangle) /\langle\tau\rangle$ with the same mean waiting time as that of the TSD with $\lambda=10^{-6}$ (triangles): $\langle\tau\rangle=c \lambda^{\alpha-1} \alpha$. The dot-dashed line is a theoretical prediction for the exponential distribution: $R(t)=\left(c \alpha \lambda^{\alpha-1} / t\right)^{1 / 2}$. Note that the scales of vertical axes of two figures are different. For comparison, the theoretical prediction of RSD for the non-equilibrium ensemble at short timescales is depicted by a dotted line in the figure (b). 
given by the time average of the following observable:

$$
\begin{aligned}
h\left(t^{\prime}\right) & =\sum_{k=1}^{\infty} \delta\left(t^{\prime}-t_{k}\right) h_{k} \\
h_{k} & =\Delta z_{k}^{2}+2 \sum_{l=1}^{k-1} z_{k} z_{l} \theta\left(\Delta-\left(t_{k}-t_{l}\right)\right),
\end{aligned}
$$

where $z_{k}= \pm 1$ is the displacement of the jump at time $t^{\prime}=t_{k}$, and $\theta(t)$ is a step function defined by

$$
\theta(t)= \begin{cases}0, & (t<0) \\ t, & (t \geq 0)\end{cases}
$$

Let us express a trajectory of a CTRW as

$$
x\left(t^{\prime}\right)=\sum_{k=1}^{\infty} z_{k} I\left(t_{k}<t^{\prime}\right)
$$

where $I\left(t_{k}<t^{\prime}\right)$ is the indicator function defined as follows: $I\left(t_{k}<t^{\prime}\right)=1$ if the inside of the bracket is satisfied, while $I\left(t_{k}<t^{\prime}\right)=0$ otherwise. Then, the displacement $x\left(t^{\prime}+\Delta\right)-x\left(t^{\prime}\right)$ is given by

$$
x\left(t^{\prime}+\Delta\right)-x\left(t^{\prime}\right)=\sum_{k=1}^{\infty} z_{k} I\left(t^{\prime}<t_{k}<t^{\prime}+\Delta\right) .
$$

Furthermore, the squared displacement $\left[x\left(t^{\prime}+\Delta\right)-x\left(t^{\prime}\right)\right]^{2}$ is expressed as

$$
\begin{aligned}
{\left[x\left(t^{\prime}+\Delta\right)-x\left(t^{\prime}\right)\right]^{2}=} & \sum_{k=1}^{\infty} I\left(t_{k}-\Delta<t^{\prime}<t_{k}\right) z_{k}^{2} \\
& +2 \sum_{k=1}^{\infty} \sum_{l=1}^{k-1} z_{k} z_{l} I\left(t_{k}-\Delta<t^{\prime}<t_{l}\right) .
\end{aligned}
$$

From Eqs. (60) and (1), we obtain the following approximation for the TAMSD:

$$
\overline{(\delta x)^{2}}(\Delta, t) \approx \frac{1}{t} \sum_{k=1}^{N_{t}}\left[\Delta z_{k}^{2}+2 \sum_{l=1}^{k-1} z_{k} z_{l} \theta\left(\Delta-\left(t_{k}-t_{l}\right)\right)\right] .
$$

Now, it is clear that the RHS of Eq. (61) is equivalent to the time average of $h\left(t^{\prime}\right)$, defined by Eqs. (55) and (56).

\section{CTRWs without reflecting boundaries}

In the absence of the confinement effect [23] (i.e., without reflecting boundaries), the relations $\left\langle h_{k}\right\rangle=\Delta$ and $\left\langle h_{k} h_{k+n}\right\rangle-\left\langle h_{k}\right\rangle\left\langle h_{k+n}\right\rangle=0$ for $n \geq 1$ hold because of the mutual independence of $z_{k},\left\langle z_{k}\right\rangle=0$ and $z_{k}^{2}=1$. In addition, since $z_{k}$ is independent of the initial ensemble, the same relations can be obtain also for the equilibrium ensemble: $\left\langle h_{k}\right\rangle_{\mathrm{eq}}=\Delta$ and $\left\langle h_{k} h_{k+n}\right\rangle_{\mathrm{eq}}-\left\langle h_{k}\right\rangle_{\mathrm{eq}}\left\langle h_{k+n}\right\rangle_{\mathrm{eq}}=$
0 . Then, $h_{k}[$ Eq. (56)] satisfies the law of large numbers $[\mathrm{Eq} \cdot(\mathrm{H} 2)]$; therefore, we have

$$
\overline{(\delta x)^{2}}(\Delta, t) \approx \frac{1}{t} \sum_{k=1}^{N_{t}} h_{k}=\frac{N_{t}}{t} \frac{1}{N_{t}} \sum_{k=1}^{N_{t}} h_{k}=\frac{N_{t}}{t} \Delta,
$$

for arbitrary $\Delta$. Note that this result is independent of the choice of the initial ensembles. Therefore, TAMSD increases in proportion to $\Delta$, and has the same statistical property as $N_{t}$. In particular, the diffusion constant $D_{t}$ is given by

$$
D_{t}=\frac{N_{t}}{t} .
$$

Furthermore, the PDF of $D_{t}$ is given by Eq. (41), and the RSD of $D_{t}$ shows the asymptotics such as the ones given in Eq. (50).

\section{CTRWs with reflecting boundaries}

Although a similar property holds for the system with reflecting boundaries, the calculation becomes more complicated, because $z_{k}(k=1,2, \ldots)$ are not mutually independent. Here, we present only an outline of the proof. The main tool is the spectral decomposition of the $n$ time transition matrix $P^{(n)}$ of the DTRWs with reflecting boundaries [43]:

$$
P^{(n)}=\sum_{r=0}^{L-1}|r\rangle\langle r| \lambda_{r}^{n}
$$

where $L$ is the total number of sites. The ket $|r\rangle$ is the $L$ dimensional eigenvector of the transition matrix defined by

$$
|0\rangle=\left(\begin{array}{c}
\frac{1}{L^{1 / 2}} \\
\vdots \\
\frac{1}{L^{1 / 2}}
\end{array}\right)
$$

for $r=0$, and

$$
|r\rangle=\left(\begin{array}{c}
r_{1} \\
\vdots \\
r_{L}
\end{array}\right) \quad \text { with } \quad r_{j}=\frac{\sin \frac{\pi r j}{L}-\sin \frac{\pi r(j-1)}{L}}{L^{1 / 2}\left(1-\cos \frac{\pi r}{L}\right)^{1 / 2}}
$$

for $1 \leq r \leq L-1$. Here, $j$ is a site index $(1 \leq j \leq L)$. Also, the bra $\langle r|$ is defined by the transpose of $|r\rangle$. The real numbers $\lambda_{r}$ are the associated eigenvalues: $\lambda_{0}=1$ for $r=0$ and $\lambda_{r}=\cos (\pi r / L)$ for $1 \leq r \leq L-1$. Thus, the first term $(r=0)$ in Eq. (64) corresponds to the eigenmode with the unit eigenvalue (the non-decaying mode), which is a uniform state, and the other terms decay exponentially fast to zero. Each element of the transition matrix, i.e., $P_{i, j}^{(n)}$, is the transition probability from the site $i$ to the site $j$ during $n$ jumps. An important point is that each element of the matrix tends to 
$1 / L$ exponentially fast as $n$ increases. It follows that correlation functions for the DTRWs decay exponentially, too. By using this fact, we can show (after some lengthy calculations) that the correlation functions for the CTRWs $\left\langle h_{k} h_{k+n}\right\rangle-\left\langle h_{k}\right\rangle\left\langle h_{k+n}\right\rangle$, which can be expressed with correlation functions for the DTRWs such as $\left\langle z_{n} z_{l} z_{k} z_{1}\right\rangle-\left\langle z_{n} z_{l}\right\rangle\left\langle z_{k} z_{1}\right\rangle$, also decay exponentially.

As a result, the law of large numbers holds for the $h_{k}$ even for the confined system, and we obtain

$$
\overline{(\delta x)^{2}}(\Delta, t) \approx \frac{N_{t}}{t} \frac{1}{N_{t}} \sum_{k=1}^{N_{t}} h_{k}=\frac{N_{t}}{t} \mu_{h}(\Delta) .
$$

The function $\mu_{h}(\Delta)$ can be derived by using the transition matrix [Eq. (64)] with a suitable hydrodynamic limit, however an easier way here is to utilize the results in Sec. III. For example, from Eqs. (31) and (47) [or Eqs. (33) and (47)] we obtain

$$
\overline{(\delta x)^{2}}(\Delta, t) \approx \begin{cases}\frac{N_{t}}{t} \Delta, & \text { for } \Delta \ll \Delta_{c} \\ \frac{N_{t}}{t} \frac{c L^{2} \Delta^{1-\alpha}}{6 \Gamma(2-\alpha)}, & \text { for } \Delta \gg \Delta_{c},\end{cases}
$$

for the non-equilibrium initial ensemble. Similarly, from Eqs. (35) and (53), we have Eq. (68) also for the equilibrium ensemble. Thus, Eq. (68) is valid for both nonequilibrium and equilibrium ensembles. Thus, all the differences between the two ensembles are included in the statistical properties of $N_{t}$ in Eq. (68). See, for example, Eqs. (47) and (53).

\section{CONCLUSION}

Up to now, two characteristic properties have been reported for confined CTRWs [38]: (i) there is a crossover from normal to anomalous diffusion, and (ii) TAMSDs are distributed depending on trajectories (i.e., weak ergodicity breaking). These results are for the nonequilibrium ensemble [There is no equilibrium ensemble for CTRWs with power-law waiting times with stable in$\operatorname{dex} \alpha \in(0,1)$, because the system never reaches an equilibrium state.]. In this paper, in addition to this confinement effect, we incorporated a cutoff into the waiting time distribution. For the case of the non-equilibrium ensemble, we analytically determined that the property (i) persists even in the long measurement time limit $t \rightarrow \infty$ (This fact was first found numerically in [2]). In contrast, the property (ii), that is, the distributional behavior of TAMSDs, appears for short measurement times $t$, whereas ergodicity holds for longer measurement times. The important point is that as compared to common distributions such as exponential distribution, it takes a very long time to observe ergodic behavior for the case where the waiting time distribution is given by the TSD. In addition, this transition from weak ergodicity breaking to ergodicity is a transition from an irreproducible regime to a reproducible regime.

Furthermore, as shown in Eq. (51), the crossover time $t_{c}$ between weak ergodicity breaking and ergodicity is proportional to $1 / \lambda$ [also, the crossover time between anomalous and normal diffusion in EAMSD for free CTRWs is proportional to $1 / \lambda$; see the text below Eq. (47)]. Because the mean waiting time $\langle\tau\rangle$ is given by $\langle\tau\rangle \sim 1 / \lambda^{1-\alpha}$, we have

$$
t_{c} \sim\langle\tau\rangle^{\frac{1}{1-\alpha}} .
$$

Thus, the crossover time $t_{c}$ is not proportional to the mean waiting time $\langle\tau\rangle$; in fact, $t_{c}$ can be much longer than $\langle\tau\rangle$ for $\alpha$ close to 1 . These facts may be important for estimating the crossover time in experiments [3].

In contrast to the CTRWs with power-law waiting times with stable index $\alpha \in(0,1)$, the CTRWs with cutoff waiting times have an equilibrium state. It is surprising that the crossover from normal to anomalous diffusion [property (i)], and a scatter in TAMSD [property (ii)] exist even for the case of the equilibrium initial ensemble. The scatter in TAMSD is even broader than the case of the non-equilibrium ensemble. The main difference from the non-equilibrium case is that there is no aging for the equilibrium ensemble. Another difference between the two ensembles is that the RSD decays algebraically even in the short time regime for the equilibrium case, whereas it does not decay for the non-equilibrium case. These properties for the equilibrium ensemble might fit well with some experimental results $[1,2,5,7]$ (See the next section). The important point is that the absence of aging in experimental data does not necessarily exclude the possibility of the CTRWs. We also presented a numerical method to generate the equilibrium ensemble (Appendix B).

\section{DISCUSSION}

First, we compare the model studied in this paper (in particular the equilibrium CTRWs) with the experimental data presented in [1]. In [1], they studied lipid granules in a harmonic potential, and used confined nonequilibrium CTRWs as a theoretical model. They presented the following quantitative agreements between the experimental data and the model: (1) the crossover from normal to anomalous diffusion in TAMSD and (2) the scatter distribution of the TAMSD. They set the total measurement time $t=3 \mathrm{sec}$ for these data, which seems to correspond to the case (B) in our classification [Eq. (8): namely, a time regime after aging]. See the Fig. 6 in the supplementary material of [1].

However, there seems to be one disagreement between the experimental data and the non-equilibrium CTRWs: i.e., (3) the aging property. In fact, the non-equilibrium CTRWs show aging in short measurement times [time regime (A) in Eq. (8)], whereas the experimental data do not (see the Fig. 6 in the supplementary material). 
On the other hand, the equilibrium CTRWs reproduce all three properties. In the time regime (B) [Eq. (8)], the equilibrium CTRWs show quantitatively the same behavior as the non-equilibrium CTRWs with respect to the properties (1) and (2). Thus, the equilibrium CTRWs will also reproduce these experimental observations successfully. In addition, the equilibrium CTRWs show no aging even in the time regime (A), and thus the model agrees the experimental data with respect to the property (3), too.

Second, although we show that time averages for a class of observables including TAMSD follow the ML distribution (Appendix H), different distributions can arise for different classes of observables [51]. As an example, we show in Appendix $\mathrm{H}$ a case in which long time averages follow the PDF called the bilateral ML distribution [52].

Third, there are qualitatively different types of random walk models in random environments. Recently, anomalous properties have been found in both experiments [1$3,5-8,53]$ and molecular dynamics simulations $[50,54]$. The CTRWs, which are a model of random walks in random environments, are often used as a model of these anomalous properties. However, there are similar random walk models in random environments with qualitatively different statistical behavior: for example, barrier models $[11,55]$, random force models $[11,56]$ and the reptation model for entangled polymers $[50,57]$. To the best of the authors' knowledge, the ergodic properties, such as the behavior of RSD, of these systems are still unclear.

Finally, besides random walks in random environments, there are still several different mechanisms for anomalous behavior, e.g., GLE, FBMs, and diffusion on fractal structures [58]. Therefore, it is important to develop techniques for time series analysis to elucidate which mechanism is the actual cause of the anomalous properties observed in various experiments and molecular dynamical simulations $[9,59]$. It is also important to investigate systems in which these mechanisms are combined [60], and those in which nonlinear dynamics are coupled with these anomalous mechanisms [21, 53].

\section{ACKNOWLEDGMENTS}

We thank S. Shinkai for drawing our attention to Ref.[52], and T. Uneyama for fruitful discussions concerning possible connections between CTRWs and entangled polymer systems. In addition, we are grateful to an anonymous referee for his helpful comments on Eq. (10) and Appendix D. This study is partially supported by a Grant-in-Aid for Young Scientists (B) (22740262).

\section{Appendix A: Infinitely divisible distribution}

Here we briefly introduce the one-sided TSD. In general, the infinitely divisible distributions $P(\tau)$ is defined as follows: if $\omega(s)$ is the characteristic function (Fourier transform) of $P(\tau)$, then there exists a characteristic function $\omega_{n}(s)$ such that $\omega(s)=\omega_{n}^{n}(s)$. Note that this is not trivial, because the function $\omega^{1 / n}(s)$ is not necessarily a characteristic function of a PDF (i.e., a non-negative function).

Now let us define the one-sided TSD $P_{\mathrm{TL}}(\tau, \lambda)$. First, we define the characteristic function $e^{\psi(\zeta, \lambda)}$ of $P_{\mathrm{TL}}(\tau, \lambda)$ as follows:

$$
\begin{aligned}
P_{\mathrm{TL}}(\tau, \lambda) & =\frac{1}{2 \pi} \int_{-\infty}^{\infty} e^{\psi(\zeta, \lambda)} e^{-i \zeta \tau} d \zeta, \\
e^{\psi(\zeta, \lambda)} & =\int_{-\infty}^{\infty} P_{\mathrm{TL}}(\tau, \lambda) e^{i \zeta \tau} d \tau .
\end{aligned}
$$

Then, the function $\psi(s, \lambda)$ is defined by the canonical form of the infinitely divisible distributions [61]:

$$
\psi(\zeta, \lambda)=\int_{-\infty}^{\infty}\left(e^{i \zeta \tau}-1\right) f(\tau, \lambda) d \tau
$$

If $f(\tau, \lambda)$ is a PDF in terms of $\tau$, then $e^{\psi(\zeta, \lambda)}$ is the characteristic function of the compound Poisson distribution [61]. For the TSD, however, the function $f(\tau, \lambda)$ is not a $\mathrm{PDF}$, and is defined as follows [22, 33, 35-37]:

$$
f(\tau, \lambda)= \begin{cases}0, & (\tau<0) \\ -c \frac{\tau^{-1-\alpha} e^{-\lambda \tau}}{\Gamma(-\alpha)}, & (\tau>0),\end{cases}
$$

where $c$ is the scale factor, and $\alpha$ and $\lambda$ are parameters satisfying $0<\alpha<1$ and $\lambda \geq 0$, respectively. By changing the integration path on the complex plane in Eq. (A3) to $C: z=s(\lambda+i \zeta) /\left(\lambda^{2}+\zeta^{2}\right)[s \in[0, \infty)]$, we obtain $\psi(\zeta, \lambda)$ as

$$
\psi(\zeta, \lambda)=-c\left[(\lambda-i \zeta)^{\alpha}-\lambda^{\alpha}\right] .
$$

The above equation indicates an important property:

$$
n \psi(\zeta, \lambda)=\psi\left(n^{1 / \alpha} \zeta, n^{1 / \alpha} \lambda\right)
$$

which is related to the infinite divisibility introduced in the first paragraph of this section, because it can be rewritten as $\psi\left(n^{-1 / \alpha} \zeta, n^{-1 / \alpha} \lambda\right)=\psi(\zeta, \lambda) / n$, and $\psi\left(n^{-1 / \alpha} \zeta, n^{-1 / \alpha} \lambda\right)$ is obviously a characteristic function. It is clear that Eq. (5) is obtained from Eq. (A6).

Now, we derive Eq. (4). From Eqs. (A1) and (A5), we have

$$
\begin{aligned}
P_{\mathrm{TL}}(\tau, \lambda) & =\frac{e^{c \lambda^{\alpha}}}{2 \pi} \operatorname{Re} \int_{0}^{\infty} e^{-c(\lambda-i \zeta)^{\alpha}} e^{-i \zeta \tau} d \zeta \\
& =\frac{e^{c \lambda^{\alpha}}}{\pi \tau} \operatorname{Im} \int_{0}^{\infty} e^{-c\left(\lambda-\frac{s}{\tau}\right)^{\alpha}} e^{-s} d s,
\end{aligned}
$$


where we have changed the integration path from the real to the imaginary axis by using the Cauchy's integral theorem. Then, we have

$$
\begin{aligned}
P_{\mathrm{TL}}(\tau, \lambda) & =\frac{e^{c \lambda^{\alpha}-\lambda \tau}}{\pi \tau} \operatorname{Im} \int_{-\tau \lambda}^{\infty} e^{-c\left(-\frac{s}{\tau}\right)^{\alpha}} e^{-s} d s \\
& =\frac{e^{c \lambda^{\alpha}-\lambda \tau}}{\pi \tau} \operatorname{Im} \int_{0}^{\infty} e^{-c\left(-\frac{s}{\tau}\right)^{\alpha}} e^{-s} d s
\end{aligned}
$$

Finally, we obtain Eq. (4) by Taylor expansion of the exponential function $e^{-c\left(-\frac{s}{\tau}\right)^{\alpha}}$ and the integral representation of the Gamma function $\Gamma(k \alpha+1)=\int_{0}^{\infty} s^{\alpha k} e^{-s} d s$.

The equilibrium waiting time distribution (7) can be derived by using Eq. (B5) as follows:

$$
P_{\mathrm{TL}}^{\mathrm{eq}}(\tau, \lambda)=\int_{0}^{1} \frac{\tau}{a\langle\tau\rangle} P_{\mathrm{TL}}\left(\frac{\tau}{a}, \lambda\right) d a .
$$

\section{Appendix B: Method of Numerical Simulation}

Let $Y_{\lambda}$ be a random variable following the TSD [Eq. (4)]. We briefly review the method to generate $Y_{\lambda}$ numerically according to [33]. First, a random variable $Y_{0}$, which follows the one-sided stable distributions, can be generated by the following equation [62]:

$$
\begin{aligned}
Y_{0}= & c^{1 / \alpha} \frac{\sin \left(\alpha\left(V+\frac{\pi}{2}\right)\right)}{(\cos V)^{1 / \alpha}} \\
& \times\left[\frac{\cos \left(V-\alpha\left(V+\frac{\pi}{2}\right)\right)}{W}\right]^{(1-\alpha) / \alpha},
\end{aligned}
$$

where $V$ is a uniform noise in $[-\pi / 2, \pi / 2]$, and $W$ is an exponential noise with mean 1 . To generate $Y_{\lambda}$, note that TSD [Eq. (4)] is just a stable distribution multiplied by the exponential factor $e^{-\lambda \tau}$. Therefore, we first generate $Y_{0}$, then we accept it with a probability $\exp \left(-\lambda Y_{0}\right)<$ 1. If $Y_{0}$ is rejected, it will be regenerated according to Eq. (B1) until it is accepted. Thereafter, the random variable that is finally accepted follows TSD [33].

Similarly, to generate equilibrium noise $Y_{\lambda}^{e q}$, first we create an auxiliary random variable $T$ by accepting $Y_{0}$ with a probability $\lambda Y_{0} \exp \left(-\lambda Y_{0}+1\right)$. Then, $Y_{\lambda}^{e q}$ is obtained by $Y_{\lambda}^{e q}=X T$, where $X$ is a uniform noise in $[0,1]$. This can be proved as follows.

First, let us consider a time axis $(-\infty, \infty)$ covered by many non-overlapping time intervals. Each interval is obtained from $P_{\mathrm{TL}}(\tau, \lambda)$ (The intervals are assumed to be mutually independent). Then, choose a time $t$ randomly from the time axis. Now, the random variable $T$ is defined by the length of the time interval which includes the randomly chosen time $t$. In addition, let $\rho(\tau)$ be the PDF of the variable $T$. Then, $\rho(\tau)$ is given by

$$
\rho(\tau)=\frac{\tau}{\langle\tau\rangle} P_{\mathrm{TL}}(\tau, \lambda) \propto \tau e^{-\lambda \tau} P_{\mathrm{TL}}(\tau, 0),
$$

because the probability with which an interval is chosen is proportional to the length of that interval $\tau$.
Thus, the random variable $T$ can be generated from the stable noise $Y_{0}$ by accepting it with the probability $\lambda Y_{0} \exp \left(-\lambda Y_{0}+1\right) \leq 1$ (The factor $\lambda e$ is added to make the maximum probability be unity for computational efficiency.). Moreover, by the Laplace transform of the above equation, we have

$$
\tilde{\rho}(s)=-\frac{1}{\langle\tau\rangle} \frac{\partial \tilde{P}_{\mathrm{TL}}}{\partial s}(s, \lambda) .
$$

Next, let us define a new variable $Y_{\lambda}^{\mathrm{eq}}=X T$, where $X$ is the uniform noise in $[0,1]$. Then, we have

$\operatorname{Prob}\left(Y_{\lambda}^{\mathrm{eq}}>\tau\right)=\operatorname{Prob}(X T>\tau)=\int_{0}^{1} \operatorname{Prob}\left(T>\frac{\tau}{a}\right) d a$.

By differentiating the both side, we obtain

$$
\rho_{\mathrm{eq}}(\tau)=\int_{0}^{1} \rho\left(\frac{\tau}{a}\right) \frac{d a}{a}
$$

where $\rho_{\text {eq }}(\tau)$ is the PDF of $Y_{\lambda}^{\mathrm{eq}}$. The Laplace transform of the above equation leads to

$$
\tilde{\rho}_{\mathrm{eq}}(s)=\int_{0}^{1} \tilde{\rho}(a s) d a .
$$

Inserting Eq. (B3) into the above equation, we obtain $\tilde{\rho}_{\text {eq }}(s)=\left[1-\tilde{P}_{\mathrm{TL}}(s, \lambda)\right] /\langle\tau\rangle s$, which is the Laplace transform of the equilibrium waiting time PDF [Eq. (6)]. Therefore, $Y_{\lambda}^{\mathrm{eq}}$ follows the equilibrium waiting time distribution $P_{\mathrm{TL}}^{\mathrm{eq}}(\tau, \lambda)$.

This method to generate the equilibrium waiting time might be applicable to other probability densities with finite moments.

\section{Appendix C: Time translation invariance of the equilibrium waiting time}

Let $w(\tau)$ be a waiting time distribution for a renewal process, and $w^{\mathrm{eq}}(\tau)$ be the associated equilibrium waiting time distribution. Here, we show the relation $w_{\mathrm{e}}^{\mathrm{eq}}\left(\tau ; t^{\prime}\right)=$ $w^{\mathrm{eq}}(\tau)[$ Eq. $(9)]$ by the method presented in [47]. First, let us define

$$
w\left(\tau ; t^{\prime}, n\right) \equiv\left\langle\delta\left(\tau-\left(t_{n+1}-t^{\prime}\right)\right) I\left(t_{n}<t^{\prime}<t_{n+1}\right)\right\rangle,
$$

where $I\left(a<t^{\prime}<b\right)=1$ if the inside of the bracket is satisfied, 0 otherwise. In addition, $t_{n}(n=1,2, \ldots)$ are renewal times defined by $t_{n} \equiv \sum_{k=1}^{n} \tau_{k}$, where $\tau_{k}$ are successive waiting times. The Laplace transform with respect to $\tau$ and $t^{\prime}$ gives

$$
\begin{aligned}
\breve{w}(u ; s, n) & =\left\langle\int_{t_{n}}^{t_{n+1}} e^{-\left(t_{n+1}-t^{\prime}\right) u} e^{-t^{\prime} s} d t^{\prime}\right\rangle \\
& = \begin{cases}\frac{\tilde{w}^{\mathrm{eq}}(u)-\tilde{w}^{\mathrm{eq}}(s)}{s-u}, & \text { for } n=0 \\
\tilde{w}^{\mathrm{eq}}(s) \tilde{w}^{n-1}(s) \frac{\tilde{w}(u)-\tilde{w}(s)}{s-u}, & \text { for } n \geq 1 .\end{cases}
\end{aligned}
$$


Because $\tilde{w}(u ; s)=\sum_{n=0}^{\infty} \tilde{w}(u ; s, n)$, we have

$$
\breve{w}_{\mathrm{e}}^{\mathrm{eq}}(u ; s)=\frac{\tilde{w}(u)-\tilde{w}(s)}{s-u} \cdot \frac{\tilde{w}^{\mathrm{eq}}(s)}{1-\tilde{w}(s)}+\frac{\tilde{w}^{\mathrm{eq}}(u)-\tilde{w}^{\mathrm{eq}}(s)}{s-u} .
$$

Here, if we replace $\tilde{w}^{\text {eq }}(s)$ with $\tilde{w}(s)$, we obtain the forward recurrence time distribution for the non-equilibrium ensemble [Eq. (16)]:

$$
\breve{w}_{\mathrm{e}}(u ; s)=\frac{\tilde{w}(u)-\tilde{w}(s)}{s-u} \cdot \frac{1}{1-\tilde{w}(s)} .
$$

It follows that

$$
\tilde{w}^{e q}(u)=\lim _{s \rightarrow 0} s \tilde{w}_{\mathrm{e}}(u ; s)=\frac{1-\tilde{w}(u)}{\langle\tau\rangle u} .
$$

By inserting Eq. (C5) into Eq. (C3), we find that $\breve{w}_{\mathrm{e}}^{\mathrm{eq}}(u ; s)=\tilde{w}^{e q}(u) / s$. The inverse Laplace transform gives the relation we want.

\section{Appendix D: Derivation of Eq. (10)}

In this appendix, we present a detailed derivation of Eq. (10), which we owe to an anonymous referee.

Here, we consider the non-equilibrium initial ensemble, i.e., $t=0$ is the renewal time (the particle jumps at $t=0)$. First, we write the LHS of Eq. (10) as

$$
\left\langle\left[x\left(t^{\prime}+\Delta\right)-x\left(t^{\prime}\right)\right]^{2}\right\rangle=\int_{-\infty}^{\infty} d x x^{2} l\left(x ; t^{\prime}, t^{\prime}+\Delta\right),
$$

where $l\left(x ; t^{\prime}, t^{\prime}+\Delta\right)$ is the PDF of the displacement in the interval $t \in\left[t^{\prime}, t^{\prime}+\Delta\right]$. Furthermore, $l\left(x ; t^{\prime}, t^{\prime}+\Delta\right)$ can be rewritten as

$$
l\left(x ; t^{\prime}, t^{\prime}+\Delta\right)=\sum_{n=0}^{\infty} P\left(n ; t^{\prime}, t^{\prime}+\Delta\right) l^{* n}(x),
$$

where $P\left(n ; t^{\prime}, t^{\prime}+\Delta\right)$ is the probability of having $n$ jumps in the interval $t \in\left[t^{\prime}, t^{\prime}+\Delta\right], l(x)$ is the PDF of single jump length, and $l^{* n}(x)$ is the $n$-times convoluted PDF of $l(x)$ with $l^{* 0}(x)=\delta(x)$. Note that we set $l(x)=[\delta(x+$ $1)+\delta(x-1)] / 2$ in our model. Moreover, the following recursion relation is the most essential ingredient:

$$
\begin{aligned}
P\left(n ; t^{\prime}, t^{\prime}+\Delta\right) & =\int_{0}^{\Delta} d \tau w_{e}\left(\tau ; t^{\prime}\right) P\left(n-1 ; t^{\prime}+\tau, t^{\prime}+\Delta\right) \\
& =\int_{0}^{\Delta} d \tau w_{e}\left(\tau ; t^{\prime}\right) P(n-1 ; 0, \Delta-\tau)
\end{aligned}
$$

for $n \geq 1$. Note that we can replace $t^{\prime}+\tau$ with 0 (and $t^{\prime}+\Delta$ with $\Delta-\tau$ ), because both $t^{\prime}+\tau$ and 0 are the renewal times.
Putting these equations together, we obtain

$$
\begin{aligned}
& \left\langle\left[x\left(t^{\prime}+\Delta\right)-x\left(t^{\prime}\right)\right]^{2}\right\rangle \\
& =\int_{0}^{\Delta} d \tau w_{e}\left(\tau ; t^{\prime}\right) \sum_{n=0}^{\infty} P(n ; 0, \Delta-\tau) \int_{-\infty}^{\infty} d x x^{2} l^{*(n+1)}(x) .
\end{aligned}
$$

Now, if we put $l(x)=[\delta(x+1)+\delta(x-1)] / 2$ into the convolution of the above equation, $l^{*(n+1)}(x)=\left[l^{* n} *\right.$ $l](x)$, we have Eq. (10).

\section{Appendix E: Derivation of GFFPE}

GFFPE was derived from a subordinated process in [33]. Here, we derive GFFPE from CTRWs in a hydrodynamic limit $[11,63,64]$. Let $P(x, t)$ be the PDF of a particle at a time $t$, and $P_{0}(x)$ be the initial density $P_{0}(x) \equiv P(x, 0)$. (In this section, we consider $t$ to be the usual time variable, instead of the total measurement time). Moreover, each particle is assumed to follow the CTRW dynamics on the real line $x$ or a one-dimensional lattice $x=x_{0}, x_{ \pm 1}, x_{ \pm 2}, \ldots$. Now, we define $\psi(x, t) d x d t$ as the probability of a particle to perform a jump with length $x$ after being trapped for a certain period $t$. Then, the probability of a particle to be trapped for a period $t$ is given by

$\phi(t)=1-\int_{-\infty}^{\infty} d x^{\prime} \int_{0}^{t} \psi\left(x^{\prime}, t^{\prime}\right) d t^{\prime}=1-\int_{0}^{t} w\left(t^{\prime}\right) d t^{\prime}$.

Furthermore, we define $Q(x, t) d t d x$ as the probability of a particle to reach an interval $[x, x+d x)$ in the period $[t, t+d t)$. Then, we have

$$
\begin{aligned}
P(x, t)= & \int_{0}^{t} d t^{\prime} \phi\left(t-t^{\prime}\right) Q\left(x, t^{\prime}\right)+\phi(t) P_{0}(x), \\
Q(x, t)= & \int_{-\infty}^{\infty} d x^{\prime} \int_{0}^{t} d t^{\prime} \psi\left(x^{\prime}, t^{\prime}\right) Q\left(x-x^{\prime}, t-t^{\prime}\right) \\
& +\int_{-\infty}^{\infty} d x^{\prime} \psi\left(x^{\prime}, t\right) P_{0}\left(x-x^{\prime}\right)
\end{aligned}
$$

Taking the Fourier and Laplace transforms with respect to space and time, respectively, we obtain

$$
\begin{aligned}
\tilde{Q}(k, u) & =\frac{\tilde{\psi}(k, u) \tilde{P}_{0}(k)}{1-\tilde{\psi}(k, u)}, \\
\tilde{P}(k, u) & =\frac{1-\tilde{w}(u)}{u} \frac{\tilde{P}_{0}(k)}{1-\tilde{\psi}(k, u)},
\end{aligned}
$$

where we used the relation $\tilde{\phi}(u)=(1-\tilde{w}(u)) / u$ [the Laplace transform of Eq. (E1)].

Next, we assume that the PDF $\psi(x, t)$ can be separable, i.e., $\psi(x, t)=l(x) w(t)$. We further assume that

$$
\tilde{l}(k) \simeq 1-\frac{\left\langle\delta x^{2}\right\rangle}{2} k^{2}
$$


in the hydrodynamic limit $k \rightarrow 0$, where $\left\langle\delta x^{2}\right\rangle$ is the mean squared displacement of a single jump, i.e., $\left\langle\delta x^{2}\right\rangle=$ $\int_{-\infty}^{\infty} d x x^{2} l(x)$. For one-dimensional CTRWs with jumps only to the nearest neighbor sites, we have $\left\langle\delta x^{2}\right\rangle=1$. Moreover, we use the TSD, $P_{\mathrm{TL}}(t)$, as the waiting time distribution $w(t)$ :

$$
\tilde{w}(u) \simeq 1-c\left[(\lambda+u)^{\alpha}-\lambda^{\alpha}\right],
$$

where $\lambda, u \ll 1$ is assumed. Under these assumptions, Eq. (E5) can be rewritten as follows:

$$
u \tilde{P}(k, u)-\tilde{P}_{0}(k) \simeq-\frac{\left\langle\delta x^{2}\right\rangle}{2 c} \frac{u k^{2}}{\left[(\lambda+u)^{\alpha}-\lambda^{\alpha}\right]} \tilde{P}(k, u) .
$$

The inverse Fourier and Laplace transformations with respect to space and time lead to

$$
\frac{\partial P(x, t)}{\partial t}=K \hat{\Phi}_{t} \frac{\partial^{2} P(x, t)}{\partial x^{2}}
$$

where $K$ is a constant given by $K=\langle\delta x\rangle^{2} / 2 c$, and $\hat{\Phi}_{t}$ is an operator defined by

$$
\hat{\Phi}_{t} f(t) \equiv \frac{d}{d t} \int_{0}^{t} M\left(t-t^{\prime}\right) f\left(t^{\prime}\right) d t^{\prime}
$$

with function $M(t)$, which is defined by its Laplace transform:

$$
\tilde{M}(u)=\int_{0}^{\infty} d t e^{-u t} M(t) d t=\frac{1}{(\lambda+u)^{\alpha}-\lambda^{\alpha}} .
$$

As $\lambda \rightarrow 0$, Eq. (E9) leads to the usual FFPE [38, 64]. Thus, the above equations (E9)- (E11) are called generalized FFPE [33]. Note also that GFFPE is the equation for non-equilibrium initial ensemble. In contrast, in order to describe the system started with the equilibrium ensemble, the multi-point fractal diffusion equation would be necessary [65].

\section{Appendix F: Mixed problem of GFFPE}

The initial-boundary value problem of GFFPE [Eq. (E9)] under the boundary condition

$$
\frac{\partial P}{\partial x}(0, t)=\frac{\partial P}{\partial x}(L, t)=0,
$$

and initial condition

$$
P(x, 0)=\delta\left(x-x_{s}\right)
$$

can be solved by a standard method for the diffusion equation [38]. In fact, by assuming the separability $P(x, t)=X(x) T(t)$, we obtain

$$
\frac{T^{\prime}(t)}{K \hat{\Phi}_{t} T(t)}=\frac{X^{\prime \prime}(x)}{X(x)}=-q,
$$

where $q$ is a constant. $X(x)$ satisfying the boundary condition $[\mathrm{Eq} .(\mathrm{F} 1)]$ is given by $X_{n}(x)=\cos (n \pi x / L)$ and $q=(n \pi / L)^{2}(n=0,1,2 \ldots)$. Similarly, $T(t)$ is derived in its Laplace form:

$$
\tilde{T}_{n}(u)=\frac{T_{n}(0)}{u+(n \pi / L)^{2} K u \tilde{M}(u)} .
$$

Then, we have the solution in the following form: $P(x, t)=\sum_{n=0}^{\infty} X_{n}(x) T_{n}(t)$. Finally, $T_{n}(0)$ in Eq. (F4) can be determined from the initial condition [Eq. (F2)] as

$$
T_{0}(0)=\frac{1}{L}, \quad T_{n}(0)=\frac{2}{L} \cos \frac{n \pi x_{s}}{L} \quad(n=1,2, \ldots) .
$$

Finally, we obtain

$$
P(x, u)=\frac{1}{L u}+\frac{2}{L} \sum_{n=1}^{\infty} \frac{\cos \frac{n \pi x_{s}}{L} \cos \frac{n \pi x}{L}}{u+(n \pi / L)^{2} K u \tilde{M}(u)} .
$$

Note that $P(x, u)$ in the above equation is just the transition probability $P\left(x, u ; x_{s}, 0\right)$ used in Sec. III.

\section{Appendix G: Riemann zeta function}

First, we define the functions $\zeta^{e}(\beta)$ and $\zeta^{o}(\beta)$ as

$$
\zeta^{e}(\beta) \equiv \sum_{\substack{n=2 \\ n \text { :even }}}^{\infty} \frac{1}{n^{\beta}}, \quad \zeta^{o}(\beta) \equiv \sum_{\substack{n=1 \\ n: \text { odd }}}^{\infty} \frac{1}{n^{\beta}} .
$$

By using the integral representation of the gamma function,

$$
\frac{1}{n^{\beta}}=\frac{1}{\Gamma(\beta)} \int_{0}^{\infty} d v v^{\beta-1} e^{-n v}
$$

it is easy to obtain the following formula:

$$
\zeta^{e}(\beta)=\frac{\zeta(\beta)}{2^{\beta}},
$$

where $\zeta(\beta)$ is the Riemann zeta function. From Eq. (G3), we have

$$
\zeta^{o}(\beta)=\frac{2^{\beta}-1}{2^{\beta}} \zeta(\beta) .
$$

In particular, we have $\zeta^{o}(4)=\pi^{4} / 96$ and $\zeta^{o}(2)=\pi^{2} / 8$.

\section{Appendix H: Ergodic theorems for general observables}

In this appendix, we summarize the ergodic properties of general observables. As an example, we derive the spatial distribution of free CTRWs. First, we consider a renewal process with renewal times $t_{k}(k=1,2, \ldots)$ (see Sec. II). We also define observables that take nonzero values only at the renewal times $t^{\prime}=t_{k}(k=1,2, \ldots)$ :

$$
h\left(t^{\prime}\right)=\sum_{k=1}^{\infty} H_{k} \delta\left(t^{\prime}-t_{k}\right)
$$


where $\left\{H_{k}\right\}$ are random variables with the same mean value $\left\langle H_{k}\right\rangle=\mu_{h}(k=1,2, \ldots)$. $\left\{H_{k}\right\}$ are assumed to be independent of $N_{t}$, but not necessarily mutually independent. We also assume the ergodicity with respect to the operational time, i.e., the number of renewals $n$ :

$$
\frac{1}{n} \sum_{k=1}^{n} H_{k} \simeq \mu_{h}, \quad \text { as } \quad n \rightarrow \infty .
$$

This relation is just the law of large numbers. A sufficient condition for Eq. (H2) is that the correlation function $C(n) \equiv\left\langle H_{k} H_{k+n}\right\rangle-\left\langle H_{k}\right\rangle\left\langle H_{k+n}\right\rangle$ decays faster than $n^{-\gamma}(\gamma>0)[11,66]$.

From Eq. (H1), the time average of the function $h\left(t^{\prime}\right)$ is given by

$$
\frac{1}{t} \int_{0}^{t} d t^{\prime} h\left(t^{\prime}\right)=\frac{1}{t} \sum_{k=1}^{N_{t}} H_{k}
$$

Note that the value of the RHS of Eq. (H3) depends on the trajectories of the renewal process in general. For example, if $H_{k} \equiv 1(k=1,2, \ldots)$ and the PDF of the renewal time $\tau$ is give by a power law $w(\tau) \sim 1 / \tau^{1+\alpha}$ with $0<\alpha<1$, the RHS of the above equation is equivalent to $N_{t} / t$, which follows the ML distribution [Eq. (41) with $\lambda=0]$ as $t \rightarrow \infty$.

First, we consider the case in which $\mu_{h} \neq 0$. In this case, we obtain the following equation from Eq. (H2):

$$
\frac{1}{N_{t}} \sum_{k=1}^{N_{t}} H_{k} \rightarrow \mu_{h}, \quad \text { as } \quad t \rightarrow \infty .
$$

Thus, Eq. (H3) can be rewritten as

$$
\frac{1}{t} \int_{0}^{t} d t^{\prime} h\left(t^{\prime}\right) \simeq \frac{N_{t}}{t} \mu_{h}
$$

Similarly, if another observable $g\left(t^{\prime}\right)$ defined by $g\left(t^{\prime}\right)=$ $\sum_{k=1}^{\infty} G_{k} \delta\left(t^{\prime}-t_{k}\right)$ satisfies the same conditions as $h\left(t^{\prime}\right)$, we have

$$
\frac{\int_{0}^{t} g\left(t^{\prime}\right) d t^{\prime}}{\int_{0}^{t} h\left(t^{\prime}\right) d t^{\prime}} \rightarrow \frac{\mu_{g}}{\mu_{h}}, \quad \text { as } \quad t \rightarrow \infty
$$

Note that the RHS is not a random variable. Equation (H6) is a stochastic version of Hopf's ergodic theorem for dynamical systems [28].

Next, we consider the case $\mu_{h}=0$. We assume that the correlation function $C(n)$ decays faster than $1 / n$; then, from the central limit theorem, we have

$$
\frac{1}{n^{1 / 2}} \sum_{k=1}^{n} H_{k} \simeq F_{\sigma}, \quad \text { as } \quad n \rightarrow \infty,
$$

where $F_{\sigma}$ is a random variable following the Gaussian distribution with mean 0 and variance $\sigma^{2}[11,67]$. The variance $\sigma^{2}$ is given by $\sigma^{2}=C(0)+2 \sum_{n=1}^{\infty} C(n)$ [11]. From Eqs. (H3) and (H7), we obtain

$$
\frac{1}{t} \int_{0}^{t} d t^{\prime} h\left(t^{\prime}\right) \simeq \frac{N_{t}^{1 / 2}}{t} F_{\sigma} .
$$

If we define a random variable $Y_{t}$ as $Y_{t} \equiv\left(N_{t} / t^{\alpha}\right)^{1 / 2} F_{\sigma}$, its characteristic function $\left\langle e^{i \xi Y_{t}}\right\rangle$ is given by

$$
\left\langle e^{i \xi Y_{t}}\right\rangle=\tilde{f}_{\lambda}\left(\frac{\sigma^{2} \xi^{2}}{2}, t\right),
$$

where $\tilde{f}_{\lambda}(\xi, t)$ is the Laplace transform of the PDF $f_{\lambda}(x, t)$ [Eq. (41)]. Although, in general, it is difficult to obtain the PDF of $Y_{t}$, we can derive it explicitly for $\lambda=0$. In this case, since $\tilde{f}_{0}(\xi)=\tilde{f}_{0}(\xi, t)$, which is the Laplace transform of the ML distribution, we obtain

$$
\tilde{f}_{0}(\xi)=\sum_{k=0}^{\infty}\left(-\frac{\xi}{c}\right)^{k} \frac{1}{\Gamma(\alpha k+1)} .
$$

To derive this equation, let us introduce an auxiliary variable $h$ in Eq. (38) as $\operatorname{Prob}\left(N_{t} / t^{\alpha}<x h\right)=$ $\int_{h x^{-1 / \alpha}}^{\infty} d \tau P_{\mathrm{TL}}(\tau, 0)$. Then double Laplace transformations with respect to $h$ and $x$ give $\frac{1}{s \xi} \sum_{k=0}^{\infty}\left(-\xi / c s^{\alpha}\right)^{k}$, where $s$ is the Laplace variable conjugate to $h$. Finally, taking the inverse Laplace transform with respect to $h$ and setting $h=1$, we obtain Eq. (H10). Here note that $\operatorname{Prob}\left(N_{t} / t^{\alpha}<x h\right)$ is the integral of the PDF $f_{0}(x, t)$. By using Eqs. (H9) and (H10), we obtain the characteristic function for $\lambda=0$ explicitly:

$$
\left\langle e^{i \xi Y_{t}}\right\rangle=\sum_{k=0}^{\infty}\left(\frac{i \xi}{\sqrt{2 c} / \sigma}\right)^{2 k} \frac{1}{\Gamma((\alpha / 2) \cdot 2 k+1)} .
$$

Note that the PDF of $Y_{t}$ is symmetric with respect to the $y$-axis from its definition and that the even order moments of $Y_{t}$ are equivalent to those of the ML distribution with index $\alpha / 2$ and scale factor $\sqrt{2 c} / \sigma$ [Eq. (H10)]. This means that the PDF of $Y_{t}, f_{0}^{b}(x)$, is a symmetric extension of the ML distribution; therefore we obtain

$f_{0}^{b}(x)=\frac{-1}{\alpha \pi} \sum_{k=1}^{\infty} \frac{\Gamma\left(\frac{k \alpha}{2}+1\right)}{k !}\left(-\frac{\sqrt{2 c}}{\sigma}\right)^{k}|x|^{k-1} \sin \left(\frac{\pi k \alpha}{2}\right)$

for $-\infty<x<\infty$. The PDF $f_{0}^{b}(x)$ is called the bilateral Mittag-Leffler distribution [52]. The simplest example of this PDF is the spatial distribution of one-dimensional free CTRWs. Let the random variable $H_{k}$ be the displacement of the $k$-th jump (e.g., $H_{k}= \pm 1$ for CTRWs with jumps only to the nearest neighbor sites). If the jumps are symmetric, then $\left\langle H_{k}\right\rangle=0$. Thus, the time average [Eq. (H8)] becomes a rescaled spatial position and the PDF [Eq. (H12)] is the spatial distribution of the free CTRWs. Note that this PDF is equivalent to the one derived from the FFPE [Eq. (46) in [64]]. 
[1] J.-H. Jeon, V. Tejedor, S. Burov, E. Barkai, C. SelhuberUnkel, K. Berg-Sørensen, L. Oddershede, and R. Metzler, Phys. Rev. Lett. 106, 048103 (2011).

[2] S. Burov, J. Jeon, R. Metzler, and E. Barkai, Phys. Chem. Chem. Phys. 13, 1800 (2011).

[3] Q. Xu, L. Feng, R. Sha, N. C. Seeman, and P. M. Chaikin, Phys. Rev. Lett. 106, 228102 (2011).

[4] I. Y. Wong, M. L. Gardel, D. R. Reichman, E. R. Weeks, M. T. Valentine, A. R. Bausch, and D. A. Weitz, Phys. Rev. Lett. 92, 178101 (2004).

[5] I. Golding and E. C. Cox, Phys. Rev. Lett. 96, 098102 (2006).

[6] S. C. Weber, A. J. Spakowitz, and J. A. Theriot, Phys. Rev. Lett. 104, 238102 (2010); S. C. Weber, J. A. Theriot, and A. J. Spakowitz, Phys. Rev. E 82, 011913 (2010).

[7] I. Bronstein, Y. Israel, E. Kepten, S. Mai, Y. Shav-Tal, E. Barkai, and Y. Garini, Phys. Rev. Lett. 103, 018102 (2009); E. Kepten, I. Bronshtein, and Y. Garini, Phys. Rev. E 83, 041919 (2011).

[8] D. S. Banks and C. Fradin, Biophys. J. 89, 2960 (2005).

[9] M. Magdziarz, A. Weron, K. Burnecki, and J. Klafter, Phys. Rev. Lett. 103, 180602 (2009); M. Magdziarz and J. Klafter, Phys. Rev. E 82, 011129 (2010).

[10] H. Scher and E. W. Montroll, Phys. Rev. B 12, 2455 (1975).

[11] J. Bouchaud and A. Georges, Phys. Rep. 195, 127 (1990).

[12] In this work, we use " " to stand for an asymptotic relation, i.e., $f(s) \sim g(s)$ means $f(s) / g(s)=\mathcal{O}(1)$ for some asymptotic limit such as $s \rightarrow 0$. On the other hand, we use " $\simeq$ " to stand for a stricter asymptotic relation: $f(s) \simeq g(s)$ means $f(s) / g(s)=1$ for some asymptotic limit. Furthermore, we use " $A \approx B$ " when $A$ is an approximation of $B$, and " $A \propto B$ " when $A$ is proportional to $B$, that is, they are not necessarily asymptotic relations.

[13] M. J. Saxton, Biophys. J. 92, 1178 (2007).

[14] J. Machta, J. Phys. A 18, L531 (1985).

[15] T. Miyaguchi and T. Akimoto, Phys. Rev. E 83, 031926 (2011).

[16] G. H. Weiss and S. Havlin, Physica A 134, 474 (1986).

[17] I. Goychuk and P. Hänggi, Proc. Natl. Acad. Sci. USA 99, 3552 (2002).

[18] N. Pottier, Physica A 317, 371 (2003).

[19] A. D. Viñales and M. A. Despósito, Phys. Rev. E 73, 016111 (2006).

[20] T. G. Mason and D. A. Weitz, Phys. Rev. Lett. 74, 1250 (1995); A. J. Levine and T. C. Lubensky, ibid. 85, 1774 (2000).

[21] W. Deng and E. Barkai, Phys. Rev. E 79, 011112 (2009); J.-H. Jeon and R. Metzler, ibid. 81, 021103 (2010); I. Goychuk, ibid. 80, 046125 (2009).

[22] M. Magdziarz and A. Weron, Ann. Phys. 326, 2431 (2011).

[23] T. Miyaguchi and T. Akimoto, Phys. Rev. E 83, 062101 (2011).

[24] J. P. Bouchaud, J. Phys. I France 2, 1705 (1992).

[25] N. Korabel and E. Barkai, Phys. Rev. E 82, 016209 (2010).

[26] Y. He, S. Burov, R. Metzler, and E. Barkai, Phys. Rev. Lett. 101, 058101 (2008).
[27] A. Lubelski, I. M. Sokolov, and J. Klafter, Phys. Rev. Lett. 100, 250602 (2008).

[28] J. Aaronson, An Introduction to Infinite Ergodic Theory (American Mathematical Society, Province, 1997).

[29] T. Akimoto and T. Miyaguchi, Phys. Rev. E 82, 030102 (2010).

[30] W. Young, A. Pumir, and Y. Pomeau, Phys. Fluids A 1, 462 (1989).

[31] C. Song, T. Koren, P. Wang, and A. Barabasi, Nat Phys 6, 818 (2010).

[32] M. V. Matthews, W. L. Ellsworth, and P. A. Reasenberg, Bull. Seism. Soc. Am. 92, 2233 (2002).

[33] J. Gajda and M. Magdziarz, Phys. Rev. E 82, 011117 (2010); 84, 021137 (2011).

[34] R. N. Mantegna and H. E. Stanley, Phys. Rev. Lett. 73, 2946 (1994).

[35] I. Koponen, Phys. Rev. E 52, 1197 (1995).

[36] H. Nakao, Phys. Lett. A 266, 282 (2000).

[37] A. Cartea and D. del Castillo-Negrete, Phys. Rev. E 76, 041105 (2007); D. del Castillo-Negrete, 79, 031120 (2009); A. Stanislavsky and K. Weron, Phys. Lett. A 373, 2520 (2009); A. Stanislavsky, K. Weron, and A. Weron, Phys. Rev. E 78, 051106 (2008).

[38] T. Neusius, I. M. Sokolov, and J. C. Smith, Phys. Rev. E 80, 011109 (2009).

[39] A. Granéli, C. C. Yeykal, R. B. Robertson, and E. C. Greene, Proc. Natl. Acad. Sci. U.S.A 103, 1221 (2006).

[40] Y. M. Wang, R. H. Austin, and E. C. Cox, Phys. Rev. Lett. 97, 048302 (2006).

[41] A. Kusumi, Y. Sako, and M. Yamamoto, Biophys. J. 65, 2021 (1993).

[42] J.-H. Jeon and R. Metzler, J. Phys. A 43, 252001 (2010).

[43] W. Feller, An Introduction to Probability Theory and its Applications, 3rd ed., Vol. I (Wiley, New York, 1968) Chap. 16; J. G. Kemeny and J. Snell, Finite Markov Chains (Springer, 1983).

[44] P. Lévy, Théory de l'Addition des Variables Aléatoires (Gauthier-Villars, Paris, 1937).

[45] W. Feller, An Introduction to Probability Theory and its Applications, 2nd ed., Vol. II (Wiley, New York, 1971) Chap. 17.

[46] D. R. Cox, Renewal theory (Methuen, London, 1962).

[47] C. Godrèche and J. M. Luck, J. Stat. Phys. 104, 489 (2001).

[48] E. Barkai and Y.-C. Cheng, J. Chem. Phys. 118, 6167 (2003); G. Margolin and E. Barkai, 121, 1566 (2004).

[49] J. H. P. Schulz, E. Barkai, and R. Metzler, Phys. Rev. Lett. 110, 020602 (2013); T. Akimoto and E. Barkai, arXiv:1209.6170 (2012).

[50] T. Akimoto, E. Yamamoto, K. Yasuoka, Y. Hirano, and M. Yasui, Phys. Rev. Lett. 107, 178103 (2011); T. Uneyama, T. Akimoto, and T. Miyaguchi, J. Chem. Phys. 137, 114903 (2012).

[51] A. Rebenshtok and E. Barkai, Phys. Rev. Lett. 99, 210601 (2007); J. Stat. Phys. 133, 565 (2008); T. Akimoto, 132, 171 (2008); A. Saa and R. Venegeroles, Phys. Rev. E 82, 031110 (2010).

[52] Y. Kasahara, Publ. RIMS, Kyoto Univ. 12, 801 (1977).

[53] T. Harada, T. Yokogawa, T. Miyaguchi, and H. Kori, Biophys. J. 96, 255 (2009).

[54] T. Neusius, I. Daidone, I. M. Sokolov, and J. C. Smith, 
Phys. Rev. E 83, 021902 (2011).

[55] S. Alexander, J. Bernasconi, W. R. Schneider, and R. Orbach, Rev. Mod. Phys. 53, 175 (1981).

[56] M. Slutsky, M. Kardar, and L. A. Mirny, Phys. Rev. E 69, 061903 (2004); M. Slutsky and L. A. Mirny, Biophys. J. 87, 4021 (2004).

[57] Doi and S. F. Edwards, J. Chem. Soc., Faraday Trans. 74, 1789 (1789).

[58] S. Havlin and D. Ben-Avraham, Advances in Physics 51, 187 (2002).

[59] M. Magdziarz and A. Weron, Phys. Rev. E 84, 051138 (2011).

[60] Y. Meroz, I. M. Sokolov, and J. Klafter, Phys. Rev. E 81, 010101 (2010).

[61] W. Feller, An Introduction to Probability Theory and its Applications, 2nd ed., Vol. II (Wiley, New York, 1971).

[62] J. M. Chambers, C. L. Mallows, and B. W. Stuck, J. Am.
Stat. Assoc. 71, 340 (1976); R. Weron, Stat. Probab. Lett. 28, 165 (1996); A. Janicki and A. Weron, Simulation and Chaotic Behavior of $\alpha$-Stable Stochastic Processes (Marcel Dekker Inc, 1993).

[63] E. W. Montroll and G. H. Weiss, J. Math. Phys. 6, 167 (1965).

[64] R. Metzler and J. Klafter, Phys. Rep. 339, 1 (2000).

[65] A. Baule and R. Friedrich, EPL (Europhy. Lett.) 77, 10002 (2007); E. Barkai and I. M. Sokolov, J. Stat. Mech. 2007, P08001 (2007); M. Politi, T. Kaizoji, and E. Scalas, EPL (Europhysics Letters) 96, 20004 (2011); M. Meerschaert and P. Straka, J. Stat. Phys. 149, 878 (2012); arXiv:1206.1960 (2012).

[66] S. Burov, R. Metzler, and E. Barkai, Proc. Natl. Acad. Sci. U.S.A 107, 13228 (2010).

[67] S.-K. Ma, Statistical Mechanics (World Scientific, Singapore, 1985). 